Annuaire suisse de politique de développement

$20 \mid 2001$

Promotion du secteur privé dans le cadre de la coopération au développement

\title{
3. Investissements directs et responsabilité sociale des entreprises
}

Jurg Bärlocher, Bastienne Joerchel Anhorn et Peter Utting

\section{CpenEdition}

\section{Journals}

Édition électronique

URL : http://journals.openedition.org/aspd/881

DOI : $10.4000 /$ aspd. 881

ISSN : 1663-9669

Éditeur

Institut de hautes études internationales et du développement

Édition imprimée

Date de publication : 1 avril 2001

Pagination : 85-132

ISSN : $1660-5934$

Référence électronique

Jurg Bärlocher, Bastienne Joerchel Anhorn et Peter Utting, « 3. Investissements directs et responsabilité sociale des entreprises », Annuaire suisse de politique de développement [En ligne], 20 | 2001, mis en ligne le 27 août 2012, consulté le 07 septembre 2020. URL : http:// journals.openedition.org/aspd/881 ; DOI : https://doi.org/10.4000/aspd.881 


\section{INVESTISSEMENTS DIRECTS ET RESPONSABILITÉ SOCIALE DES ENTREPRISES}

\subsection{INVESTISSEMENTS DIRECTS SUISSES DANS LES PAYS EN DÉVELOPPEMENT, EN VOIE D'INDUSTRIALISATION ET EN TRANSITION}

\section{JÜRG BÄRLOCHER*}

\section{INTRODUCTION}

Investissements directs et politique de développement: de quelles données disposons-nous pour analyser ce sujet? Notre présentation passe en revue les bases auxquelles se réfère la statistique des investissements directs, puis expose les résultats qui concernent la Suisse. Elle part d'une description des normes élaborées par des organisations internationales afin d'harmoniser les données nationales en la matière. Puis viennent quelques explications sur la statistique suisse; et enfin les chiffres concrets de la période 1993-1999, l'accent étant mis sur les investissements suisses dans les pays en développement, en voie d'industrialisation et en transition.

\section{OBJET DE LA STATISTIQUE DES INVESTISSEMENTS DIRECTS}

La statistique suisse sur les investissements directs se réfère au Balance of Payments Manual du FMI (BOPM) ${ }^{1}$ et à la Définition de référence de l'OCDE des investissements directs internationaux (Benchmark Definition of Foreign Direct Investment $)^{2}$. Le BOPM contient des normes concernant la manière d'établir la balance des paiements et la statistique des avoirs extérieurs. La Définition de référence reprend les critères définis par le BOPM, mais décrit de façon plus précise comment les données relatives aux investissements directs doivent être enregistrées pour satisfaire aux normes internationales.

La balance des paiements fait apparaitre les investissements directs comme un élément du compte des mouvements de capitaux, qui répertorie ces mouvements entre sujets économiques indigènes et étrangers. Dans la statistique des avoirs extérieurs, les investissements directs constituent une partie des créances et des engagements indigènes à l'égard de l'étranger. Il convient de souligner ici deux aspects: tout d'abord le fait que les investissements directs sont des placements d'avoirs et qu'ils se distinguent ainsi des investissements au sens de la comptabilité nationale (maintien ou accroissement de l'appareil de production); ensuite, le fait que le terme d'investissements directs peut désigner aussi bien des transactions que des portefeuilles.

\footnotetext{
* Collaborateur scientifique à la Banque nationale suisse.

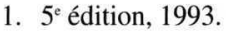

2. $3^{\mathrm{e}}$ édition, 1996.
} 


\section{$\square$ Quand y a-t-il une relation d'investissement direct?}

Selon le BOPM et la Définition de référence, les investissements directs sont des placements internationaux destinés à exercer une influence durable sur l'activité d'entreprises situées à l'étranger. Tandis que quand on a affaire à la catégorie des investissements de portefeuille (dans la balance des paiements et la statistique des avoirs extérieurs), c'est l'obtention d'un rendement approprié qui prime. La statistique opère la délimitation entre ces catégories en examinant la part du capital-actions que détient l'investisseur. D'après les normes que se sont données le FMI et l'OCDE, il y a relation d'investissement direct lorsqu'un investisseur indigène possède $10 \%$ ou plus du capital donnant droit de vote d'une société sise à l'étranger. Cette limite de $10 \%$ est jusqu'à un certain point arbitraire; ce qui est important, c'est de ne pas confondre l'influence qui peut s'exercer sur la conduite d'une entreprise avec le contrôle de celle-ci (part de suffrages dépassant $50 \%$ ).

\section{$\square$ Quels sont les agrégats?}

Selon les règles adoptées par les organisations internationales, sont considérés comme investissements directs aussi bien les transactions initiales établissant ce type de relations que tous les mouvements de capitaux ultérieurs entre investisseurs et entreprises bénéficiant de ces investissements directs. On distingue ici les éléments suivants:

- acquisition ou vente de participations aux fonds propres (y compris sous forme d'échanges d'actions);

- bénéfices réinvestis;

- octroi et remboursement de crédits (crédits au sein de groupes).

Les bénéfices réinvestis représentent des transactions fictives, en ce sens que l'on considère ces gains comme un revenu payé à l'investisseur et immédiatement réinvesti. Dans la statistique des capitaux, ces produits apparaissent avec les participations aux fonds propres.

\section{LA STATISTIQUE SUISSE DES INVESTISSEMENTS DIRECTS}

La Banque nationale suisse tient une statistique des investissements directs depuis le début des années 60. Mais elle n'était assujettie à aucune obligation d'informer avant 1993, ce qui a influencé la statistique à deux égards: en premier lieu, celle-ci n'était pas complète; mais comme toutes les entreprises multinationales ayant leur siège social en Suisse y ont participé d'emblée, la qualité des données en a moins souffert pour les investissements directs suisses à l'étranger que pour les investissements directs étrangers en Suisse. Deuxièmement, certaines sociétés ont demandé que les données publiées n'entrent pas dans les détails. La loi sur la statistique fédérale entrée en vigueur en 1993 a instauré le relevé obligatoire pour les entreprises ayant des capitaux d'investissement direct supérieurs à 10 millions de francs. Cette amélioration des bases juridiques a permis de rendre l'enquête plus complète. Seule la protection des données restreint désormais les informations publiées, lesquelles sont censées ne rien révéler de la situation d'entreprises particulières. On se conforme à cette règle en ne publiant un agrégat que s'il porte sur au moins cinq déclarations durant une période suffisamment longue et sans domination d'investissements particuliers. 
Le relevé des investissements directs ventile les flux et les volumes de capitaux dans les catégories évoquées plus haut, à savoir participations aux fonds propres, bénéfices réinvestis et crédits au sein de groupes. Les volumes indiqués correspondent à des valeurs comptables. La Définition de référence recommande en principe de baser les évaluations sur des valeurs marchandes, mais accepte pour des raisons pratiques le système adopté par de nombreux pays de répertorier les volumes des capitaux d'investissement direct d'après leur valeur comptable.

\section{$\square$ Effectif du personnel}

En plus des capitaux investis, la statistique relève l'effectif du personnel dans les entreprises où ont lieu les investissements directs. L'effectif annoncé doit être proportionnel à la part du capital de l'entreprise considérée. Un investisseur qui détient $20 \%$ du capital d'une société qui occupe 1000 personnes indiquera donc un effectif de 200 personnes.

\section{$\square$ Répartition par pays}

Dans le cas de participations indirectes, par exemple quand un investisseur participe à l'entreprise 1 du pays $\mathrm{A}$, laquelle détient elle-même une participation à l'entreprise 2 du pays B, la question se pose de savoir à quel pays destinataire attribuer l'investissement. Pour les volumes d'investissements directs à l'étranger, on applique dans la mesure du possible le principe du bénéficiaire final. Dans le cas de l'exemple ci-dessus, cela signifie que la part du capital investi dans l'entreprise 1 qui a servi à financer la participation à l'entreprise 2 sera attribuée non pas au pays A, mais au pays B. De même, la statistique attribuera les bénéfices réinvestis au pays où ils restent. Par contre, le principe du bénéficiaire final n'est guère applicable aux flux de participations aux fonds propres et de crédits. En d'autres termes, les transactions entre filiales ne sont pas enregistrées; il en résulte que le volume d'investissements directs dans un pays donné peut changer sans faire apparaître des mouvements de capitaux correspondants.

\section{$\square$ Répartition par secteurs et par branches}

Pour les investissements directs à l'étranger, cette répartition se réfère à l'activité principale de l'investisseur en Suisse. Cela peut donner lieu à certains problèmes d'interprétation pour la catégorie des sociétés financières et holdings. En règle générale, les investissements directs de groupes dont le siège social est en Suisse n'appartiennent pas à cette catégorie, même s'ils sont détenus par une société holding, du fait que ces participations ne constituent pas leur activité principale en Suisse. Divers groupes étrangers ont en revanche pour activité principale des participations étrangères en Suisse, raison pour laquelle la catégorie des sociétés financières et holdings répertorie principalement des investissements directs de groupes étrangers.

\section{INVESTISSEMENTS DIRECTS SUISSES À L'ÉTRANGER 1993-1999}

Les tableaux 1 à 3 (voir page 92 et suivantes) présentent les investissements directs suisses dans les pays en développement et en transition d'Europe centrale et orientale (dont les pays européens de la CEI), les autres pays en 
développement européens, ainsi que dans les pays en développement et nouvellement industrialisés d'Asie, d'Amérique latine et d'Afrique. Ces régions englobent les pays qui bénéficient de soutiens financiers du programme CAD de l'OCDE. Apparaissent également les investissements dans les pays de l'UE et de l'AELE, ainsi que dans les pays industrialisés du reste du monde (Etats-Unis, Canada, Japon, Australie et Nouvelle-Zélande). La somme de toutes ces entités géographiques correspond au total des investissements directs suisses à l'étranger $^{3}$. La période examinée commence en 1993 du fait que, comme nous l'avons indiqué sous le point 3, c'est à partir de cette année-là que les résultats de la statistique des investissements directs peuvent donner lieu à une publication détaillée. Les résultats antérieurs à 1993 ont été présentés dans l'Annuaire Suisse-Tiers Monde 1993.

Le tableau 1 résume les exportations de capitaux durant la période 1993-1999, ainsi que le volume des capitaux d'investissement direct et l'effectif du personnel à fin 1999. Outre les résultats par régions, ce tableau donne aussi les chiffres d'un certain nombre de pays choisis en fonction du volume des capitaux et de l'effectif du personnel, et compte tenu du nombre minimum de déclarations requis pour autoriser la publication. Alors que le tableau 1 présente les investissements à l'étranger de toutes les entreprises suisses, le tableau 2 répartit les investisseurs par secteurs. Le tableau 3, enfin, fait la distinction entre participations aux fonds propres, bénéfices réinvestis et crédits.

Les exportations de capitaux en vue d'investissements directs suisses à l'étranger ont totalisé 13 milliards de francs en 1993, et ce flux avait doublé en 1997. La part à destination des pays en développement, en voie d'industrialisation et en transition représente environ $15 \%$ des capitaux exportés au cours de la période 1993-1999. Mais les investissements dans les pays industrialisés ont provisoirement chuté en 1998 (de 6 milliards pour s'établir à 15 milliards de francs); tandis que ceux à destination des autres régions - surtout pays émergents d'Asie et d'Amérique latine et centres financiers d'Amérique centrale ont progressé la même année de 7 à 12 milliards de francs. C'est ainsi qu'en 1998 la part des pays en développement, en voie d'industrialisation ou en transition a atteint $45 \%$ des capitaux exportés à titre d'investissements directs, mais pour retomber ensuite. Les flux de capitaux vers des régions autres que les pays industrialisés ont progressé en 1999 de 1,5 milliard pour s'établir à 13,5 milliards de francs; ils n'ont donc pas suivi la forte progression des investissements dans les pays industrialisés, lesquels ont fait cette année-là un saut qui les a portés de 15 à 41 milliards de francs.

Les investissements directs suisses à l'étranger représentaient en 1999 un volume de 308 milliards de francs, dont un quart dans les pays en développement, en voie d'industrialisation et en transition. La part de ceux-ci était un peu plus élevée pour le nombre d'emplois: plus de $30 \%$ du personnel d'entreprises suisses à l'étranger travaillaient dans ces régions.

3. Un exposé des investissements directs assorti de tableaux détaillés est présenté chaque année dans le Bulletin trimestriel de la BNS (numéro 4). Une partie de ces données figure également dans le Bulletin mensuel de statistiques économiques de la BNS. Ces publications peuvent être consultées sous www.snb.ch. 


\section{$\square$ Europe centrale et orientale}

De 1993 à 1997, les investissements directs à destination des pays d'Europe centrale et orientale ont totalisé en moyenne 0,4 milliard de francs par année, principalement sous forme de participations aux fonds propres. Ces exportations de capitaux ont dépassé la barre du milliard de francs en 1998 et en 1999, avec une progression des crédits au sein de groupes et des bénéfices réinvestis.

Le volume des investissements directs dans ces pays se chiffrait à 6,1 milliards de francs à fin 1999. Les investisseurs industriels détenaient $40 \%$ de ces capitaux, ceux du tertiaire $35 \%$, les sociétés financières et holdings $25 \%$, et $60 \%$ des 72'000 emplois relevaient de participations à l'étranger du secteur industriel.

La Pologne et la République tchèque se mettent en évidence côté exportations de capitaux aussi bien que pour le volume des capitaux investis et l'effectif du personnel. De même, la Hongrie est depuis assez longtemps une destination importante d'investissements directs suisses. Croatie, Slovénie et Bulgarie ont vu le volume des capitaux investis progresser modérément mais sans interruption depuis 1993. Il a fallu par contre attendre 1997 pour que des investissements appréciables soient effectués en Slovaquie, dans les Etats baltes et en Roumanie. Les entreprises suisses ont beaucoup investi dans la Fédération russe, surtout en 1998 et 1999. Parmi les autres pays européens de la CEI, l'Ukraine a enregistré depuis 1997 un certain afflux de capitaux, tandis qu'aucun investissement d'une certaine ampleur n'a encore eu lieu en Biélorussie et en Moldavie.

\section{$\square$ Autres pays en développement européens}

Dans ce groupe, ce sont surtout les investissements directs en Turquie ainsi qu'à Chypre et à Gibraltar qui revêtent de l'importance. La Turquie se place au premier rang avec quelque $10^{\prime} 000$ emplois qui se répartissent à parts approximativement égales entre le secteur industriel et celui des services. Après une période de développement assez régulier, les exportations de capitaux dans ce pays ont brusquement augmenté en 1998; puis un mouvement inverse a eu lieu en 1999. Pour l'ensemble du groupe en revanche, on constate un solde négatif en 1998 et un solde positif pour 1999 en matière d'exportations de capitaux; ce qui est dû à des fluctuations importantes des bénéfices réinvestis pour quelques investissements directs dans des entreprises de services à Chypre et à Gibraltar.

\section{$\square$ Pays en développement et nouvellement industrialisés d'Asie}

Les pays émergents d'Asie constituent, avec les centres financiers d'Amérique centrale, les principaux sites d'investissements directs suisses en dehors des pays industrialisés. En 1993, les exportations de capitaux dans cette région ont été de 0,7 milliard de francs. Sauf en 1995 où des capitaux ont été retirés de Hongkong, ce flux financier a constamment progressé pour atteindre 5,7 milliards de francs en 1998 - un chiffre qui n'a pas tout à fait été atteint l'année suivante. Les bénéfices réinvestis ont joué ici un rôle considérable puisqu'ils représentent une bonne moitié des capitaux exportés en 1998 et 1999.

Le volume de nos investissements directs dans les pays émergents d'Asie atteignait 20,7 milliards de francs à fin 1999. C'est à Hongkong qu'il était le plus important jusqu'en 1996, et Singapour occupe le premier rang depuis lors. 
Hongkong et Singapour totalisaient ensemble tout juste 16 milliards de francs en capitaux suisses investis à fin 1999, dont quelque $70 \%$ sous forme de participations à l'étranger de sociétés financières et holdings. Les investissements suisses aux Philippines, en Malaisie, en Corée du Sud, en Thaïlande et à Taiwan se chiffraient fin 1999 à 4,7 milliards de francs, dont deux tiers dans le secteur industriel. Ces cinq pays totalisaient $70 \%$ des 102 ' 400 emplois de la région, de sorte que leur part de l'effectif du personnel était nettement supérieure à celle des capitaux.

Les exportations de capitaux suisses vers le Proche-Orient ont atteint en moyenne 0,1 milliard de francs par année entre 1993 et 1999, avec Israël et l'Arabie saoudite pour principales destinations. Et le volume des capitaux investis dans la région se chiffrait à 1,1 milliard de francs en 1999, dont deux tiers détenus par des investisseurs industriels.

Encore limités à 0,1 milliard de francs en 1993, les capitaux exportés sous forme d'investissements directs dans les autres pays en développement asiatiques ont ensuite progressé pour atteindre 1 milliard en 1996 - un volume comparable à celui des investissements dans les pays émergents d'Asie. Ces investissements ont d'abord pris le chemin de la Chine, puis l'Inde et l'Indonésie ont gagné en importance. Tandis que les flux financiers continuaient d'augmenter après 1996 vers les pays nouvellement industrialisés, on constate un recul des exportations de capitaux dans les pays en développement: l'afflux des participations aux fonds propres a passé de 0,8 milliard de francs en 1996 à 0,3 milliard en 1999. Les pertes importantes essuyées par des entreprises industrielles en Chine ont même entraîné en 1999 une réduction globale des capitaux investis dans les pays en développement d'Asie.

Le volume des investissements directs suisses dans les pays en développement asiatiques (sans le Proche-Orient) était de 3 milliards de francs en 1999, dont $70 \%$ dans le secteur industriel. Une petite moitié de ces investissements concernait la Chine, tandis que l'Inde et l'Indonésie totalisaient ensemble 0,9 milliard de francs. Les autres se situaient principalement au Pakistan, au Vietnam, au Sri Lanka et au Bangladesh, auxquels se sont ajoutés le Myanmar en 1997 et le Kazakhstan en 1999.

\section{$\square$ Amérique latine}

Les exportations de capitaux sous forme d'investissements directs dans les pays latino-américains émergents se chiffrent en moyenne à 0,2 milliard de francs entre 1993 et 1997, mais ont dépassé le milliard par année en 1998 et 1999. Le volume des capitaux investis était de 11 milliards de francs en 1999, dont $45 \%$ au Brésil, 35\% au Mexique, $10 \%$ en Argentine et autant au Chili. Les capitaux d'origine industrielle ont dominé jusqu'en 1996. En 1997 et 1998, l'industrie a momentanément retiré des capitaux de ces pays, tandis que le secteur des services (sociétés financières et holdings comprises) accroissait ses investissements en 1998 et 1999. La part du secondaire dans le volume des capitaux investis a ainsi régressé de $75 \%$ fin 1996 à tout juste $60 \%$ en 1999.

La majeure partie des capitaux directement investis dans les pays en développement latino-américains ont été dirigés sur les places financières extraterritoriales (centres offshore) de la région. Ces capitaux totalisaient 26 milliards de francs à fin 1999, pour l'essentiel aux Bermudes, aux îles Caïmans et dans les Antilles 
néerlandaises ${ }^{4}$. Les principaux investisseurs sont des entreprises de services, mais le secteur secondaire et les sociétés financières ou holdings y détiennent également des participations importantes. Les flux de capitaux avec cette région sont soumis à de grands fluctuations: cela allait entre 1993 et 1999 de retraits totalisant 0,9 milliard de francs (1994) à des exportations de capitaux atteignant 4,6 milliards (1999).

Si l'on met de côté les places financières extraterritoriales, les exportations de capitaux vers les pays en développement latino-américains ont été en moyenne d'à peine 0,2 milliard de francs entre 1993 et 1996, puis de plus de 0,6 milliard de 1997 à 1999. Fin 1999, le volume des investissements directs se chiffrait à 3,6 milliards de francs et les effectifs à 35'200 personnes. Environ la moitié de ce volume correspondait à des investissements en Colombie et au Venezuela. Il y avait des participations suisses d'une certaine importance en Uruguay et en Equateur côté capitaux investis, et aussi au Pérou, au Costa Rica et au Guatemala côté effectif du personnel. Comme pour les pays émergents, les investissements du tertiaire dans les pays en développement prennent de l'ampleur depuis 1997 ; mais les investissements directs d'entreprises industrielles représentaient encore trois quarts du total à fin 1999.

\section{Afrique}

Les exportations de capitaux vers l'Afrique étaient en moyenne de tout juste 0,2 milliard de francs entre 1993 et 1996, pour atteindre ensuite 0,3 milliard par année. C'est ainsi que durant la période de 1993 à 1999, seuls 4\% des investissements directs destinés aux pays en développement, en voie d'industrialisation et en transition ont pris le chemin du continent africain. Le volume de ces investissements se chiffrait à 3,9 milliards de francs à fin 1999 , dont 0,8 milliard en Afrique du Nord (surtout Egypte et Maroc). Des 3,1 milliards restants, 1,3 milliard était investi en Afrique du Sud. Une bonne moitié des investissements directs en Afrique du Sud est d'origine industrielle, un quart provient du tertiaire et le dernier quart des sociétés financières et holdings. Les entreprises sudafricaines bénéficiaires de ces investissements occupaient plus de 20 '000 personnes à fin 1999. Nigeria, Botswana, Namibie, Côte d'Ivoire, Tanzanie, Kenya, Cameroun et Zimbabwe totalisaient en outre 21'000 emplois. Le volume des capitaux investis dans ces pays totalisait environ 1 milliard de francs. Le Liberia et l'île Maurice, deux pays axés sur les activités financières «offshore», avaient ensemble 0,8 milliard en investissements directs suisses pour un effectif inférieur à 100 personnes.

\section{$\square$ Remarque finale}

Les résultats de la statistique des investissements directs tels qu'ils sont présentés dans ce chapitre n'ont bien entendu pas un caractère absolu ou définitif, et laissent ouvertes différentes questions relatives aux participations suisses dans les pays en développement, en voie d'industrialisation et en transition. Nous espérons toutefois avoir évoqué utilement des aspects qui, associés à l'exposé des méthodes présenté sous les points 2 et 3, contribueront à éclaircir certains problèmes spécifiques.

4. Centres financiers «offshore» selon la nomenclature d'Eurostat: Bahamas, Barbade, Bermudes, îles Vierges britanniques, Jamaïque, îles Caïmans, Montserrat, Antilles néerlandaises, Panama, Saint Kitts et Nevis. 
Tableau 1: Répartition géographique des investissements directs suisses à l'étranger par pays et effectif du personnel

\begin{tabular}{|c|c|c|c|c|c|c|c|c|c|}
\hline \multirow[t]{2}{*}{ Région / Pays } & \multirow[b]{2}{*}{1993} & \multicolumn{4}{|c|}{$\begin{array}{l}\text { Flux d'investissements } \\
\text { (en millions de francs) }\end{array}$} & \multirow[b]{2}{*}{1998} & \multirow[b]{2}{*}{1999} & \multirow{2}{*}{$\begin{array}{c}\text { Stock d'in- } \\
\text { vestissements } \\
\text { (millions de fr.) } \\
\text { fin } 1999\end{array}$} & \multirow{2}{*}{$\begin{array}{c}\text { Nombre } \\
\text { d'employés } \\
\text { (arrondi) } \\
\text { fin } 1999 \\
\end{array}$} \\
\hline & & 1994 & 1995 & 1996 & 1997 & & & & \\
\hline UE et AELE & 3290 & 8047 & 6251 & 12576 & 12776 & 10061 & 25234 & 148436 & 774500 \\
\hline Autres pays industrialisés & 6079 & 5837 & 6583 & 4421 & 8306 & 5165 & 15266 & 81063 & 336200 \\
\hline Europe centrale et orientale & 162 & 334 & 901 & 361 & 215 & 1175 & 1141 & 6139 & 71900 \\
\hline dont: Croatie & 6 & 14 & 4 & 5 & 4 & 70 & -44 & 158 & 2200 \\
\hline Pologne & 38 & 159 & 236 & 113 & 173 & 208 & 478 & 1665 & 20000 \\
\hline Fédération de Russie & -8 & 56 & 37 & 100 & 46 & 335 & 599 & 1198 & 9700 \\
\hline Slovaquie & 0 & 0 & 3 & -2 & 13 & 21 & 40 & 153 & 3600 \\
\hline République tchèque & 54 & 34 & 573 & 61 & -19 & 356 & -72 & 1630 & 14900 \\
\hline Hongrie & 34 & 52 & 31 & 62 & -35 & -76 & 80 & 851 & 9700 \\
\hline Reste de l'Europe & 32 & 24 & 121 & 49 & 314 & -449 & 734 & 2721 & 10800 \\
\hline dont: Turquie & 41 & -8 & 96 & 0 & 51 & 296 & -95 & 1013 & 10100 \\
\hline Pays nouvellement industrialisés d'Asie & 710 & 760 & -242 & 1146 & 2383 & 5689 & 4662 & 20691 & 102400 \\
\hline dont: Hongkong & 300 & 420 & -547 & 21 & 236 & 447 & 648 & 2187 & 14200 \\
\hline Corée du Sud & 12 & 29 & 19 & 66 & -57 & 283 & 179 & 1040 & 4700 \\
\hline Malaisie & 104 & 8 & 17 & 233 & 82 & 102 & 229 & 937 & 12800 \\
\hline Philippines & 74 & 14 & -8 & 39 & 50 & 1169 & 57 & 1433 & 13100 \\
\hline Singapour & 127 & 110 & 175 & 542 & 1879 & 3173 & 3543 & 13738 & 19400 \\
\hline Taïwan & 70 & 93 & 61 & 22 & 45 & 121 & 89 & 644 & 9900 \\
\hline Thaillande & 23 & 86 & 41 & 224 & 148 & 395 & -82 & 712 & 28200 \\
\hline
\end{tabular}




\begin{tabular}{|c|c|c|c|c|c|c|c|c|c|}
\hline Proche-Orient & 13 & 49 & 31 & 258 & 30 & 36 & 100 & 1112 & 11800 \\
\hline dont: Israël & 30 & 12 & 0 & 261 & -26 & 51 & 26 & 479 & 5300 \\
\hline Liban & -27 & 3 & 9 & 8 & 2 & -2 & -13 & 120 & 900 \\
\hline Arabie saoudite & 2 & 33 & 4 & 3 & 43 & -2 & 60 & 323 & 2900 \\
\hline Emirats arabes unis & 7 & 10 & 3 & 3 & 12 & 11 & 19 & 143 & 1200 \\
\hline Autres pays en développement d'Asie & 127 & 198 & 428 & 976 & 599 & 404 & -39 & 2974 & 82400 \\
\hline dont: Chine & 32 & 168 & 342 & 378 & 255 & 123 & -116 & 1387 & 37100 \\
\hline Inde & 56 & 31 & 13 & 370 & 160 & 29 & -53 & 524 & 19400 \\
\hline Indonésie & 21 & 15 & 46 & 177 & 134 & 90 & 58 & 377 & 10000 \\
\hline Pakistan & 3 & -17 & 23 & 22 & 34 & -17 & 7 & 192 & 4300 \\
\hline Vietnam & 0 & -1 & 2 & 25 & 21 & -2 & -18 & 131 & 5100 \\
\hline
\end{tabular}

\section{Pays nouvellement industrialisés}

\begin{tabular}{|c|c|c|c|c|c|c|c|c|c|}
\hline d'Amérique centrale et du Sud & 582 & 103 & 186 & 260 & -207 & 1243 & 1310 & 11034 & 122900 \\
\hline dont: Argentine & 46 & 47 & 28 & 65 & -10 & 321 & -99 & 1317 & 13000 \\
\hline Brésil & 308 & -54 & 127 & -10 & -338 & 446 & 853 & 5043 & 72000 \\
\hline Chili & 106 & 27 & 35 & 83 & 29 & -178 & 7 & 762 & 10100 \\
\hline Mexique & 122 & 84 & -3 & 121 & 111 & 654 & 548 & 3911 & 27800 \\
\hline
\end{tabular}

\section{Pays en développement}

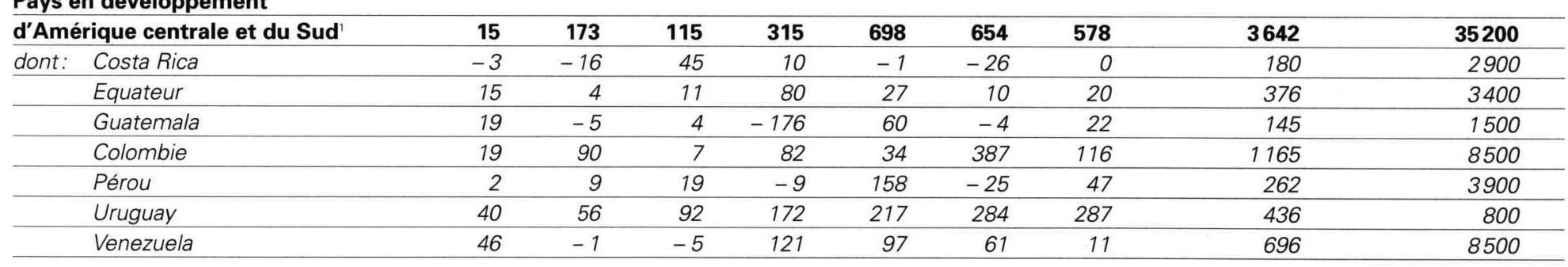


Tableau 1 : Répartition géographique des investissements directs suisses à l'étranger par pays et effectif du personnel (suite)

\begin{tabular}{|c|c|c|c|c|c|c|c|c|c|}
\hline \multirow[t]{2}{*}{ Région / Pays } & \multicolumn{7}{|c|}{$\begin{array}{l}\text { Flux d'investissements } \\
\text { (en millions de francs) }\end{array}$} & \multirow{2}{*}{$\begin{array}{c}\text { Stock d'in- } \\
\text { vestissements } \\
\text { (millions de fr.) } \\
\text { fin } 1999\end{array}$} & \multirow{2}{*}{$\begin{array}{l}\text { Nombre } \\
\text { d'employés } \\
\text { (arrondi) } \\
\text { fin } 1999\end{array}$} \\
\hline & 1993 & 1994 & 1995 & 1996 & 1997 & 1998 & 1999 & & \\
\hline \multicolumn{10}{|c|}{ Centres financiers offshore } \\
\hline d'Amérique centrale & 1876 & -864 & -113 & -583 & 236 & 2991 & 4589 & 26057 & 6500 \\
\hline Afrique du Nord & 19 & 41 & 51 & 41 & 128 & 47 & 95 & 823 & 13700 \\
\hline dont: Egypte & 12 & 33 & 34 & 18 & 92 & 57 & 93 & 472 & 8400 \\
\hline Maroc & 6 & -1 & 8 & 19 & 32 & -12 & 3 & 317 & 3500 \\
\hline Reste de I'Afrique & 43 & 60 & 125 & 142 & 256 & 194 & 336 & 3108 & 45400 \\
\hline dont: Côte d'voire & -10 & -6 & 6 & 17 & 16 & 51 & -28 & 110 & 2200 \\
\hline Nigeria & 6 & 14 & -2 & 1 & -9 & 5 & -6 & 36 & 5500 \\
\hline Afrique du Sud & 37 & 48 & 151 & 93 & 154 & 138 & 140 & 1315 & 20600 \\
\hline Ensemble des pays & 12949 & 14761 & 14438 & 19964 & 25734 & 27209 & 54005 & 307798 & 1613600 \\
\hline
\end{tabular}

1. Sans les centres financiers offshore d'Amérique centrale. 
Tableau 2: Investissements directs suisses à l'étranger, par secteurs

\begin{tabular}{|c|c|c|c|c|c|c|c|c|c|}
\hline \multirow[t]{2}{*}{ Région / Secteur } & \multirow[b]{2}{*}{1993} & \multicolumn{4}{|c|}{$\begin{array}{l}\text { Flux d'investissements } \\
\text { (en millions de francs) }\end{array}$} & \multirow[b]{2}{*}{1998} & \multirow[b]{2}{*}{1999} & \multirow{2}{*}{$\begin{array}{c}\text { Stock d'in- } \\
\text { vestissements } \\
\text { (millions de fr.) } \\
\text { fin } 1999 \\
\end{array}$} & \multirow{2}{*}{$\begin{array}{l}\text { Nombre } \\
\text { d'employés } \\
\text { (arrondi) } \\
\text { fin } 1999 \\
\end{array}$} \\
\hline & & 1994 & 1995 & 1996 & 1997 & & & & \\
\hline \multicolumn{10}{|l|}{ UE et AELE } \\
\hline Toutes les entreprises & 3290 & 8047 & 6251 & 12576 & 12776 & 10061 & 25234 & 148436 & 774500 \\
\hline Industrie & 2606 & 3438 & 3448 & 4967 & 6036 & 3322 & 3897 & 52229 & 433000 \\
\hline Services' & 1524 & 3927 & 2442 & 5549 & 2563 & 4666 & 14600 & 52828 & 175100 \\
\hline Sociétés financières et holdings & -840 & 682 & 361 & 2060 & 4178 & 2074 & 6737 & 43379 & 166300 \\
\hline \multicolumn{10}{|l|}{ Autres pays industrialisés } \\
\hline Toutes les entreprises & 6079 & 5837 & 6583 & 4421 & 8306 & 5165 & 15266 & 81063 & 336200 \\
\hline Industrie & 1560 & 4566 & 5778 & 2091 & 7381 & 686 & 4401 & 26182 & 187800 \\
\hline Services $^{1}$ & 1974 & 231 & 869 & 2185 & 260 & 3716 & 8765 & 46280 & 87600 \\
\hline Sociétés financières et holdings & 2545 & 1041 & -63 & 145 & 665 & 763 & 2099 & 8601 & 60800 \\
\hline \multicolumn{10}{|l|}{ Europe centrale et orientale } \\
\hline Toutes les entreprises & 162 & 334 & 901 & 361 & 215 & 1175 & 1141 & 6139 & 71900 \\
\hline Industrie & 95 & 192 & 231 & 197 & 151 & 422 & 540 & 2439 & 45400 \\
\hline Services ${ }^{7}$ & 56 & 85 & 524 & 89 & 99 & 421 & 155 & 2142 & 10000 \\
\hline Sociétés financières et holdings & 11 & 57 & 147 & 76 & -35 & 332 & 446 & 1557 & 16500 \\
\hline \multicolumn{10}{|l|}{ Reste de I'Europe } \\
\hline Toutes les entreprises & 32 & 24 & 121 & 49 & 314 & -449 & 734 & 2721 & 10800 \\
\hline Industrie & 15 & -21 & 53 & -26 & 38 & 117 & 6 & 480 & 4700 \\
\hline Services $^{1}$ & 11 & 36 & 55 & 70 & 289 & -683 & 530 & 1743 & 4300 \\
\hline Sociétés financières et holdings & 6 & 9 & 13 & 5 & -13 & 118 & 198 & 497 & 1900 \\
\hline
\end{tabular}


Tableau 2: Investissements directs suisses à l'étranger, par secteurs (suite)

\begin{tabular}{|c|c|c|c|c|c|c|c|c|c|}
\hline \multirow[t]{2}{*}{ Région / Secteur } & \multicolumn{7}{|c|}{$\begin{array}{l}\text { Flux d'investissements } \\
\text { (en millions de francs) }\end{array}$} & \multirow{2}{*}{$\begin{array}{c}\text { Stock d'in- } \\
\text { vestissements } \\
\text { (millions de fr.) } \\
\text { fin } 1999\end{array}$} & \multirow{2}{*}{$\begin{array}{l}\text { Nombre } \\
\text { d'employés } \\
\text { (arrondi) } \\
\text { fin } 1999\end{array}$} \\
\hline & 1993 & 1994 & 1995 & 1996 & 1997 & 1998 & 1999 & & \\
\hline \multicolumn{10}{|l|}{ Pays nouvellement industrialisés d'Asie } \\
\hline Toutes les entreprises & 710 & 760 & -242 & 1146 & 2383 & 5689 & 4662 & 20691 & 102400 \\
\hline Industrie & 158 & 271 & 41 & 218 & 435 & 3365 & 166 & 5290 & 56100 \\
\hline Services ${ }^{\prime}$ & 143 & -124 & 69 & 541 & 171 & 579 & 1868 & 3144 & 27800 \\
\hline Sociétés financières et holdings & 409 & 613 & -351 & 388 & 1777 & 1745 & 2628 & 12257 & 18500 \\
\hline \multicolumn{10}{|l|}{ Proche-Orient } \\
\hline Toutes les entreprises & 13 & 49 & 31 & 258 & 30 & 36 & 100 & 1112 & 11800 \\
\hline Industrie & 32 & 19 & 19 & 268 & 1 & 82 & 69 & 779 & 9500 \\
\hline Services $^{\prime}$ & -19 & 30 & 11 & -31 & 8 & -41 & 29 & 304 & 2100 \\
\hline Sociétés financières et holdings & 1 & 0 & 1 & 21 & 22 & -5 & 2 & 29 & 200 \\
\hline \multicolumn{10}{|l|}{ Autres pays en développement d'Asie } \\
\hline Toutes les entreprises & 127 & 198 & 428 & 976 & 599 & 404 & -39 & 2974 & 82400 \\
\hline Industrie & 104 & 174 & 322 & 488 & 463 & 88 & -223 & 2031 & 66500 \\
\hline Services $^{1}$ & 22 & 21 & 92 & 421 & 82 & 193 & 118 & 422 & 8800 \\
\hline Sociétés financières et holdings & 1 & 2 & 14 & 68 & 53 & 124 & 66 & 521 & 7100 \\
\hline \multicolumn{10}{|c|}{ Pays nouvellement industrialisés d'Amérique centrale et du Sud } \\
\hline Toutes les entreprises & 582 & 103 & 186 & 260 & -207 & 1243 & 1310 & 11034 & 122900 \\
\hline Industrie & 570 & 418 & 85 & 241 & -123 & -768 & 993 & 6503 & 75500 \\
\hline Services $^{1}$ & 15 & 12 & 70 & 85 & 79 & 1034 & 179 & 1735 & 13100 \\
\hline Sociétés financières et holdings & -3 & -327 & 31 & -66 & -163 & 977 & 138 & 2796 & 34300 \\
\hline
\end{tabular}


Pays en développement d'Amérique centrale et du Sud ${ }^{2}$

\begin{tabular}{|c|c|c|c|c|c|c|c|c|c|}
\hline Toutes les entreprises & 15 & 173 & 115 & 315 & 698 & 654 & 578 & 3642 & 35200 \\
\hline Industrie & 117 & 89 & 69 & 224 & 521 & 430 & 330 & 2737 & 20900 \\
\hline Services ${ }^{1}$ & 26 & 46 & 0 & 58 & 144 & 4 & 185 & 603 & 5600 \\
\hline Sociétés financières et holdings & -128 & 38 & 46 & 33 & 33 & 219 & 63 & 302 & 8700 \\
\hline
\end{tabular}

\begin{tabular}{|c|c|c|c|c|c|c|c|c|c|}
\hline \multicolumn{10}{|c|}{ Centres financiers offshore d'Amérique centrale } \\
\hline Toutes les entreprises & 1876 & -864 & -113 & -583 & 236 & 2991 & 4589 & 26057 & 6500 \\
\hline Industrie & 1561 & 908 & -1310 & -220 & 718 & 1671 & 594 & 4482 & 3400 \\
\hline Services ${ }^{1}$ & 635 & -18 & 1460 & 308 & 873 & 1106 & 3281 & 15641 & 1400 \\
\hline Sociétés financières et holdings & -320 & -1754 & -263 & -672 & -1355 & 214 & 713 & 5934 & 1700 \\
\hline
\end{tabular}

\section{Afrique du Nord}

\begin{tabular}{|c|c|c|c|c|c|c|c|c|c|}
\hline Toutes les entreprises & 19 & 41 & 51 & 41 & 128 & 47 & 95 & 823 & 13700 \\
\hline Industrie & 13 & 24 & 34 & 41 & 80 & 44 & 55 & 480 & 10800 \\
\hline Services' & 5 & 17 & 16 & 2 & 46 & 30 & 23 & 321 & 2600 \\
\hline Sociétés financières et holdings & 1 & 0 & 1 & -2 & 2 & -28 & 17 & 22 & 200 \\
\hline
\end{tabular}

\section{Reste de I'Afrique}

\begin{tabular}{|c|c|c|c|c|c|c|c|c|c|}
\hline & & & & & & & & & \\
\hline Toutes les entreprises & 43 & 60 & 125 & 142 & 256 & 194 & 336 & 3108 & 45400 \\
\hline Industrie & -17 & -35 & 69 & 63 & 103 & 183 & -160 & 925 & 20900 \\
\hline Services $^{1}$ & 48 & 76 & 31 & 48 & 122 & -15 & 359 & 787 & 7800 \\
\hline Sociétés financières et holdings & 11 & 19 & 25 & 31 & 32 & 26 & 137 & 1396 & 16700 \\
\hline
\end{tabular}

\section{Ensemble des pays}

\begin{tabular}{|c|c|c|c|c|c|c|c|c|c|}
\hline Ensemble des pays & & & & & & & & & \\
\hline Toutes les entreprises & 12949 & 14761 & 14438 & 19964 & 25734 & 27209 & 54005 & 307798 & 1613600 \\
\hline Industrie & 6814 & 10043 & 8838 & 8551 & 15804 & 9640 & 10667 & 104557 & 934500 \\
\hline Services ${ }^{\prime}$ & 4440 & 4338 & 5638 & 9326 & 4735 & 11010 & 30093 & 125949 & 346200 \\
\hline Sociétés financières et holdings & 1695 & 380 & -38 & 2087 & 5195 & 6559 & 13245 & 77293 & 332800 \\
\hline
\end{tabular}

6) 1. Sans sociétés financières et holdings.

2. Sans centres financiers offshore d'Amérique centrale. 
Tableau 3 : Investissements directs suisses à l'étranger, par types d'investissement

\begin{tabular}{|c|c|c|c|c|c|c|c|c|}
\hline \multirow[b]{2}{*}{ Région / Type d'investissements } & \multicolumn{5}{|c|}{$\begin{array}{l}\text { Flux d'investissements } \\
\text { (en millions de francs) }\end{array}$} & \multirow[b]{2}{*}{1998} & \multirow[b]{2}{*}{1999} & \multirow{2}{*}{ 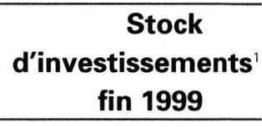 } \\
\hline & 1993 & 1994 & 1995 & 1996 & 1997 & & & \\
\hline \multicolumn{9}{|l|}{ UE et AELE } \\
\hline Total & 3290 & 8047 & 6251 & 12576 & 12776 & 10061 & 25234 & 148436 \\
\hline Participation aux fonds propres & 2634 & 7662 & 3846 & 8739 & 7688 & 4331 & 16623 & 131318 \\
\hline Bénéfices réinvestis & 1311 & 787 & 1261 & 3813 & 5177 & 4201 & 5139 & \\
\hline Crédits au sein du groupe & -655 & -403 & 1143 & 24 & -89 & 1529 & 3473 & 17118 \\
\hline \multicolumn{9}{|l|}{ Autres pays industrialisés } \\
\hline Total & 6079 & 5837 & 6583 & 4421 & 8306 & 5165 & 15266 & 81063 \\
\hline Participation aux fonds propres & 2617 & 5300 & 3670 & 3179 & 2500 & 1553 & 6178 & 62617 \\
\hline Bénéfices réinvestis & 1363 & 1178 & 1641 & 1264 & 1646 & 3099 & 5556 & \\
\hline Crédits au sein du groupe & 2099 & -640 & 1272 & -22 & 4160 & 512 & 3532 & 18446 \\
\hline
\end{tabular}

\section{Europe centrale et orientale}

\begin{tabular}{lrrrrrrrr}
\hline Total & 162 & 334 & 901 & 361 & 215 & 1175 & 1141 & 6139 \\
\hline Participation aux fonds propres & 112 & 251 & 925 & 179 & 226 & 766 & 540 & 4942 \\
\hline Bénéfices réinvestis & 8 & 13 & -53 & 89 & -32 & -244 & 358 \\
\hline Crédits au sein du groupe & 43 & 70 & 29 & 93 & 21 & 653 & 243 & \\
\hline
\end{tabular}

\section{Reste de l'Europe \\ Total}

Participation aux fonds propres

Bénéfices réinvestis

32
-5
12

$\begin{array}{rrr}24 & 121 & 49 \\ 34 & 72 & 45 \\ 2 & 28 & 51 \\ -12 & 21 & -47\end{array}$

49
45
51
-47

$\begin{array}{rrr}314 & -449 & 734 \\ 193 & 385 & 288 \\ 108 & -956 & 375 \\ 13 & 122 & 71\end{array}$

34
288
375
71




\section{Pays nouvellement industrialisés d'Asie}

\begin{tabular}{lrrrrrrrr}
\hline Total & 710 & 760 & -242 & 1146 & 2383 & 5689 & 4662 & 20691 \\
\hline Participation aux fonds propres & 355 & -60 & 80 & 865 & 796 & 3861 & 1648 \\
\hline Bénéfices réinvestis & 362 & 254 & 198 & 481 & 1355 & 1794 & 3613 \\
\hline Crédits au sein du groupe & -7 & 566 & -519 & -200 & 232 & 33 & -599 \\
\hline
\end{tabular}

\begin{tabular}{|c|c|c|c|c|c|c|c|c|}
\hline \multicolumn{9}{|l|}{ Proche-Orient } \\
\hline Total & 13 & 49 & 31 & 258 & 30 & 36 & 100 & 1112 \\
\hline Participation aux fonds propres & -31 & 27 & 19 & 246 & 45 & 59 & 34 & 1019 \\
\hline Bénéfices réinvestis & 27 & 20 & 22 & -4 & 6 & -34 & 34 & \\
\hline Crédits au sein du groupe & 17 & 2 & -10 & 16 & -20 & 11 & 32 & 93 \\
\hline
\end{tabular}

\begin{tabular}{|c|c|c|c|c|c|c|c|c|}
\hline \multicolumn{9}{|l|}{ Autres pays en développement d'Asie } \\
\hline Total & 127 & 198 & 428 & 976 & 599 & 404 & -39 & 2974 \\
\hline Participation aux fonds propres & 83 & 71 & 412 & 801 & 589 & 406 & 251 & 2367 \\
\hline Bénéfices réinvestis & 28 & 33 & -25 & 28 & -98 & -169 & -298 & \\
\hline Crédits au sein du groupe & 16 & 93 & 41 & 148 & 108 & 167 & 8 & 607 \\
\hline
\end{tabular}

\begin{tabular}{lrrrrrrr}
\hline Pays nouvellement industrialisés d'Amérique centrale et du Sud & \\
\hline Total & 582 & 103 & 186 & 260 & -207 & 1243 & 1310 \\
\hline Participation aux fonds propres & 325 & -475 & 118 & -54 & -232 & 1465 & 791 \\
\hline Bénéfices réinvestis & 230 & 547 & 92 & -36 & -12 & -437 & 316 \\
\hline Crédits au sein du groupe & -101 & 31 & -23 & 350 & 37 & 215 & 203 \\
\hline
\end{tabular}

\section{Pays en développement d'Amérique centrale et du Sud ${ }^{2}$}

\begin{tabular}{lrrrrrrrr}
\hline Pays en développement d'Amérique centrale et du Sud & & & & \\
\hline Total & 15 & 173 & 115 & 315 & 698 & 654 & 578 \\
\hline Participation aux fonds propres & 57 & 46 & 83 & -193 & 75 & 366 & 169 \\
\hline Bénéfices réinvestis & 76 & 114 & 120 & 240 & 409 & 237 & 451 \\
\hline Crédits au sein du groupe & 10 & 13 & -88 & 268 & 214 & 50 & -42 & 3246 \\
\hline
\end{tabular}


Tableau 3: Investissements directs suisses à l'étranger, par types d'investissement (suite)

\begin{tabular}{|c|c|c|c|c|c|c|c|c|}
\hline \multirow{2}{*}{ Région / Type d'investissements } & \multicolumn{5}{|c|}{$\begin{array}{l}\text { Flux d'investissements } \\
\text { (en millions de francs) }\end{array}$} & \multirow[b]{2}{*}{1998} & \multirow[b]{2}{*}{1999} & \multirow{2}{*}{$\begin{array}{c}\text { Stock } \\
\text { d'investissements' }^{\prime} \\
\text { fin } 1999 \\
\end{array}$} \\
\hline & 1993 & 1994 & 1995 & 1996 & 1997 & & & \\
\hline \multicolumn{9}{|c|}{ Centres financiers offshore d'Amérique centrale } \\
\hline Total & 1876 & -864 & -113 & -583 & 236 & 2991 & 4589 & 26057 \\
\hline Participation aux fonds propres & 831 & -1695 & 315 & -751 & 1531 & 3780 & 713 & 25202 \\
\hline Bénéfices réinvestis & 1576 & 476 & 384 & 452 & 1142 & -383 & 2631 & \\
\hline Crédits au sein du groupe & -530 & 355 & -812 & -284 & -2436 & -405 & 1246 & 855 \\
\hline
\end{tabular}

\section{Afrique du Nord}

\begin{tabular}{lrrrrrrrr}
\hline Total & 19 & 41 & 51 & 41 & 128 & 47 & 95 & 823 \\
\hline Participation aux fonds propres & 44 & 27 & 31 & 24 & 28 & -22 & 25 & 702 \\
\hline Bénéfices réinvestis & -23 & 11 & 15 & 21 & 75 & 52 & 63 \\
\hline Crédits au sein du groupe & -2 & 3 & 6 & -3 & 25 & 16 & 7 & 121
\end{tabular}

\section{Reste de I'Afrique}

\begin{tabular}{lrrrrrrrr}
\hline Total & 43 & 60 & 125 & 142 & 256 & 194 & 336 & 3108 \\
\hline Participation aux fonds propres & 57 & 55 & 68 & 26 & 76 & -3 & 389 & 26 \\
\hline Bénéfices réinvestis & -22 & 23 & 29 & 66 & 114 & 42 & 237 \\
\hline Crédits au sein du groupe & 8 & -17 & 28 & 50 & 66 & 155 & -290 & 441
\end{tabular}

\section{Ensemble des pays}

\begin{tabular}{lrrrrrrrr}
\hline Total & 12949 & 14761 & 14438 & 19964 & 25734 & 27209 & 54005 & 307798 \\
\hline Participation aux fonds propres & 7109 & 11244 & 9638 & 13106 & 13515 & 16949 & 27648 & \\
\hline Bénéfices réinvestis & 4931 & 3457 & 3711 & 6465 & 9889 & 7203 & 18474 \\
\hline Crédits au sein du groupe & 909 & 61 & 1089 & 392 & 2331 & 3058 & 7883 \\
\hline
\end{tabular}

1. Participation aux fonds propres y compris bénéfices réinvestis.

2. Sans centres financiers offshore d'Amérique centrale. 


\subsection{LES «PRINCIPES DIRECTEURS POUR MULTINATIONALES DE L'ORGANISATION DE COOPÉRATION ET DÉVELOPPEMENT ÉCONOMIQUES (OCDE)"}

BASTIENNE Joerchel ANHORN*

Le 27 juin 2000, 29 pays membres de l'OCDE, ainsi que l'Argentine, le Brésil, le Chili et la Slovaquie, ont adopté un ensemble révisé de principes directeurs à l'intention des entreprises multinationales. Ces principes sont des recommandations non contraignantes adressées par les gouvernements aux entreprises multinationales opérant dans ou depuis les pays y ayant souscrit. Il s'agit jusqu'à aujourd'hui du seul code de conduite pour entreprises multinationales relativement complet, approuvé au niveau multilatéral, et que les gouvernements s'engagent à promouvoir. Il a pour objectif d'encourager les entreprises multinationales à agir de façon responsable du point de vue social et environnemental et dans le respect des lois nationales et internationales en vigueur. Ils couvrent un large éventail de sujets relatifs aux activités des multinationales: emploi, environnement, publication d'informations, concurrence, fiscalité, science et technologie. Une référence aux droits de l'homme ainsi que deux nouveaux sujets, la lutte contre la corruption et la protection des consommateurs, ont été nouvellement intégrés.

La révision des Principes directeurs reflète les grandes mutations politiques et économiques de ces deux dernières décennies caractérisées par la mondialisation et la libéralisation des échanges internationaux. Elle répond jusqu'à un certain point aux préoccupations des pays du Sud, de la société civile et des organisations non gouvernementales $(\mathrm{ONG})$ à l'égard des déséquilibres qui caractérisent les droits et obligations des entreprises multinationales toujours plus puissantes sur le marché mondial. Elle marque aussi un tournant en confirmant l'accession des ONG de développement, d'environnement et de consommateurs sur la scène des négociations économiques internationales. Le processus de révision, mené par l'OCDE, est en effet un modèle de transparence et de démocratie puisque les ONG ont été invitées à participer activement aux différentes étapes de la négociation.

L'utilité et l'efficacité d'un instrument non contraignant suscitent toutefois un certain nombre d'interrogations dans un contexte où seules les lois du marché, de la rentabilité économique et de la compétition semblent s'imposer. Cet article a pour objectif de revenir sur le déroulement de la révision pour ensuite mettre en exergue les points forts et les points faibles des modifications apportées aux Principes directeurs. Le but est également de présenter la position des ONG qui ont participé aux négociations en se regroupant dans le cadre d'un réseau européen dont l'action s'est avérée particulièrement efficace.

\section{DÉVELOPPEMENT HISTORIQUE}

Les Principes directeurs font partie intégrante de la Déclaration de 1976 de l'OCDE sur l'investissement international et les entreprises multinationales. Cette déclaration, adoptée il y a plus de vingt ans dans un contexte économique

* Communauté de travail Swissaid, Action de Carême, Pain pour le prochain, Helvetas, Caritas, 20 octobre 2000 . 
et politique bien différent d'aujourd'hui, visait à clarifier le rôle des investissements dans l'économie mondiale et l'impact des multinationales sur les économies nationales. Les années 1970 ont en effet été marquées par l'émergence des entreprises transnationales et une internationalisation croissante de la production. Mais, à cette époque, il était d'usage que les pouvoirs publics interviennent encore fréquemment dans l'économie. Les mesures de contrôle de l'entrée et de l'activité des entreprises étrangères sur le marché national étaient très répandues.

Avec la Déclaration de 1976, les membres de 1'OCDE marquaient une double volonté. D'une part, celle de renforcer les pouvoirs des gouvernements vis-à-vis des multinationales. C'est-à-dire d'assurer le respect des législations nationales et la soumission aux autorités étatiques des entreprises de plus en plus puissantes financièrement et dont la structure internationale leur permettait d'échapper aux contrôles étatiques. D'autre part, les gouvernements, conscients du rôle stimulant que pouvaient jouer les investissements directs étrangers, souhaitaient instaurer un climat international favorable à ces derniers et régi par les principes de l'équité et de la non-discrimination ${ }^{5}$.

Les mutations politiques et économiques des années 80 et 90 ont depuis lors fortement modifié la situation. Les libéralisations et déréglementations ont conduit à l'abaissement des barrières nationales et à l'élimination des entraves et contrôles limitant l'entrée d'investissements directs étrangers et les activités des multinationales. Les échanges de biens et services ont plus que triplé durant ces deux décennies. Selon la CNUCED, les investissements directs étrangers atteindront en l'an 2000 un nouveau record de 1000 milliards de dollars ${ }^{6}$. Ils s'élevaient déjà à 865 milliards de dollars en 1999, contre 349 milliards de dollars en 1997 et 53 milliards de dollars en 1982. Ces investissements trouvent leur origine pour $80 \%$ dans les fusions et acquisitions opérées par les grands groupes transnationaux, dont neuf sur dix ont leur siège dans un pays industrialisé d'Europe ou d'Amérique du Nord.

C'est dans ce nouveau contexte qu'ont eu lieu les négociations de l' «Uruguay Round" qui ont abouti, en 1995, à la création de l'Organisation mondiale du commerce (OMC). Le but de cette organisation, regroupant aujourd'hui 138 pays membres, est la libéralisation progressive du commerce mondial et sa réglementation. Il est ancré dans la conviction, partagée encore aujourd'hui par la grande majorité de l'élite politique et économique du monde industrialisé, que seul un marché mondial entièrement libre de toute entrave peut contribuer à la croissance et au développement économique et social de la planète. L'OMC a représenté un bond en avant par rapport au GATT (General Agreement on Tariffs and Trade). D'abord, son domaine d'application s'est largement étendu, couvrant non seulement les marchandises mais aussi les services, la propriété intellectuelle, les textiles et l'agriculture. Ensuite, les règles se sont renforcées, notamment avec le système de règlement des différends et la possibilité d'appliquer des sanctions.

5. «Les "Principes directeurs" de l'OCDE dans un monde en voie de mondialisation», Arghyrios A. Fatouros, version révisée d'un document présenté par l'auteur à la Conférence de Budapest sur les «Principes directeurs» de l'OCDE, novembre 1998, publié par la Direction des affaires financières, fiscales et des entreprises de l'OCDE.

6. Rapport mondial sur l'investissement 2000, CNUCED, 4 octobre 2000. 
Les pays industrialisés ont tenté, sans succès, d'inclure les investissements dans les accords de l'OMC. Face au refus de la majorité des pays en développement, ils se sont rabattus sur 1'OCDE, où ils ont lancé, dès 1995, de nouvelles négociations pour la conclusion d'un Accord multilatéral sur l'investissement (AMI). L'idée était de négocier plus rapidement «entre gens du même monde». L'accord aurait ensuite été ouvert aux autres pays. Mais, loin d'être efficace, cette négociation à l'abri des critiques s'est avérée un obstacle fatal au moment de la conclusion finale. Les ONG de développement, d'environnement, de protection des consommateurs ainsi que les syndicats ont en effet dénoncé avec virulence le caractère unilatéral de l'AMI, qui se limitait à garantir des droits aux multinationales - en leur offrant une véritable carte blanche en matière d'investissements - sans leur imposer d'obligations, notamment environnementales et sociales.

Les Principes directeurs, que les pays de l'OCDE avaient prévu d'annexer à l'accord, se sont alors révélés largement insuffisants pour contrebalancer les nouvelles prérogatives offertes aux multinationales. En particulier, les chapitres consacrés aux questions sociales et environnementales étaient loin de répondre aux préoccupations de la société civile. Les investisseurs n'étaient notamment pas tenus de respecter les normes écologiques et sociales internationalement reconnues telles que celles de l'Organisation internationale du travail (OIT) sur les droits syndicaux ou sur le travail des enfants ou encore celles énoncées dans les divers accords multilatéraux sur l'environnement. Des questions importantes, telles que le développement durable ou la corruption, n'étaient pas même mentionnées. Et finalement, le caractère non contraignant des principes soulevait de vives critiques de la part des ONG.

Les pressions de la société civile, une résolution virulente du Parlement européen ainsi que le volte-face à la dernière minute de la France ont signé l'arrêt de mort de l'AMI en décembre 1998. Face à cet échec, les pays de l'OCDE ont rapidement décidé de poursuivre activement la révision des Principes directeurs, amorcée quelques mois plus tôt.

\section{LA RÉVISION DES PRINCIPES DIRECTEURS}

Les Principes directeurs ont subi des adaptations périodiquement. Des révisions ont notamment eu lieu en 1979, 1984 et 1991, année durant laquelle, par exemple, un chapitre sur la protection de l'environnement a été ajouté. Avec les négociations sur l'AMI, les Principes directeurs sont apparus soudainement sous les feux de la rampe et ont suscité un intérêt du public dont ils n'avaient jamais bénéficié jusque-là. De ce fait, leur révision a pris une dimension relativement inattendue pour la majorité des membres de l'OCDE, qui se seraient probablement satisfaits d'une adaptation plus minimaliste. La Suisse, par exemple, dans une prise de position datant de septembre 1998, estime que «des modifications de fond ne s'imposent pas $»^{7}$. Elle se limite à proposer, à l'instar de l'Angleterre, une adaptation des chapitres relatifs à l'environnement et à l'emploi «aux développements intervenus ces dernières années». Seule la France défend une position plus ambitieuse en invitant, en juin 1998, le CIME

7. «Réexamen des "Principes directeurs" à l'intention des entreprises multinationales», position présentée par la délégation suisse à l'OCDE, septembre 1998, page 2. 
(Comité de l'investissement international et des entreprises multinationales), responsable de la gestion des Principes directeurs à l'OCDE, «à réfléchir sur la possibilité de rendre juridiquement contraignantes certaines des dispositions des "Principes directeurs" $\gg$. Cette évolution lui semblait logique pour contrebalancer les dispositions obligatoires en matière de libéralisation des investissements prévues dans le projet de l'AMI.

Ce n'est donc finalement qu'après l'échec de l'AMI que les membres de l'OCDE se rendent à l'évidence qu'une révision de fond s'impose afin d'adapter les principes au nouveau contexte de la globalisation et montrer ainsi leur bonne volonté face à la société civile et aux ONG. On assiste donc, courant 1999, à une réflexion générale sur la nécessité de renforcer les chapitres consacrés à l'emploi et à l'environnement. De nouveaux sujets tels que la corruption et la protection des consommateurs s'imposent progressivement.

De plus, l'OCDE et ses membres marquent une claire volonté de venir à la rencontre des préoccupations de la société civile en garantissant une transparence maximale du processus de réexamen. Dès le printemps 1999, les ONG sont même activement associées aux différentes étapes de la révision. A cet effet, elles mettent sur pied une plate-forme informelle de coordination au niveau européen et délèguent des représentants pour défendre leurs points de vue à plusieurs rencontres organisées par l'OCDE. Les deux comités consultatifs officiels de l'organisation, le BIAC (Comité consultatif économique et industriel) et le TUAC (Commission syndicale consultative) participent également à ces consultations. Les projets de révision sont en outre placés régulièrement sur la page Internet de l'OCDE avec l'invitation faite au public de faire des commentaires.

En Suisse, le Secrétariat d'Etat à l'économie (Seco), en charge du dossier, utilise les réunions du groupe de liaison «Investissement» pour informer et consulter les milieux économiques et sociaux. Le groupe de liaison réunit entre autres les représentants de l'Union suisse du commerce et de l'industrie, du Groupement de holdings industrielles suisses (représentant les multinationales suisses), de l'Association suisse des banques, de l'Union syndicale suisse, de l'Union des syndicats chrétiens ainsi que d'autres départements de l'administration fédérale. La Communauté de travail Swissaid/Action de Carême/Pain pour le prochain/Helvetas/ Caritas est la seule organisation de développement invitée à ces rencontres depuis 1996. C'est également dans le cadre de ce groupe, que le Seco organise une consultation formelle de l'avant-projet des Principes directeurs au début de l'année 2000, à laquelle la Communauté de travail répondra par une prise de position détaillée, calquée sur celle des ONG actives au niveau européen.

Cette procédure de révision ouverte et flexible a débouché sur des négociations très vives entre les différents acteurs impliqués dans la révision. La version finale, approuvée en juin 2000 , reflète non seulement les conflits d'intérêt entre le secteur privé et les ONG, mais surtout aussi les différences en terme de pouvoirs économique et politique. D'un côté, les nombreuses caractéristiques des Principes directeurs ont été maintenues - en particulier leur caractère non contraignant, la structure institutionnelle ainsi que les procédures de mise en œuvre, qui restent pratiquement inchangées. De l'autre, des progrès significatifs

8. «Examen des "Principes directeurs" de l'OCDE à l'intention des entreprises multinationales, présentation des vues préliminaires de la France à l'occasion de la réunion du CIME», juin 1998. 
ont été accomplis avec l'introduction de dispositions sur les principes fondamentaux de l'homme au travail tels qu'ils sont reconnus par l'OIT: liberté syndicale et droit de représentation, interdiction du travail forcé, abolition du travail des enfants, non-discrimination. Les nouveaux chapitres sur la corruption et la protection des consommateurs, ainsi que la reconnaissance du principe de précaution dans le domaine de l'environnement et de la santé publique contribuent également à l'amélioration substantielle des Principes directeurs.

\section{LES PRINCIPALES MODIFICATIONS}

Dans la préface et le premier chapitre consacré aux «concepts et principes », les modifications portent principalement sur une description du nouveau contexte international dans lequel évoluent aujourd'hui les multinationales. Le texte reconnaît explicitement que ces dernières ont également une responsabilité à promouvoir le développement durable. Le progrès majeur réside toutefois dans l'extension géographique du champ d'application des principes à tous les pays du monde dans lesquels les multinationales exercent leurs activités. Cette revendication était formulée depuis longtemps par les ONG et les syndicats. En effet, les principes s'adressent à des multinationales, qui, par définition, mènent des activités souvent bien au-delà des frontières des pays de l'OCDE. Il devenait urgent de s'assurer que celles-ci se comportent de la même façon partout dans le monde, en particulier dans les pays en développement.

Dans le chapitre 2 consacré aux «Principes généraux», le concept du développement durable est cette fois ancré comme un objectif que les multinationales doivent viser en contribuant «aux progrès économiques, sociaux et environnementaux». Nouveaux sont aussi la référence explicite au respect des droits de l'homme ainsi qu'une disposition «not lowering standard». Celle-ci demande aux multinationales de ne pas subordonner un investissement dans un pays à l'obtention d'exemptions dans l'application de la loi en vigueur en matière d'environnement, de protection sociale, de santé, de relations avec les salariés et de fiscalité. C'est un pas important vers la reconnaissance d'une responsabilité globale des multinationales à l'égard des pays dans lesquels elles exercent leurs activités.

Le chapitre 3 sur la «publication d'informations» a été adapté aux nouvelles directives de 1'OCDE sur la «Corporate Governance»". Celles-ci exhortent les multinationales à diffuser régulièrement des informations fiables et pertinentes concernant leurs activités, leur structure, leur situation financière et leurs résultats. De plus, elles sont aussi encouragées à communiquer des informations supplémentaires sur la politique sociale, éthique et environnementale de l'entreprise. En revanche, elles bénéficient toujours d'une clause d'exception dans la mesure où elles peuvent invoquer les «coûts, la confidentialité des affaires et d'autres considérations de concurrence» pour se soustraire à l'obligation de transparence.

Le chapitre 4 sur «l'emploi et les relations professionnelles», l'un des plus importants des Principes directeurs, a été amélioré de façon substantielle. Avec l'adjonction des recommandations relatives à l'abolition du travail des enfants et du travail forcé, toutes les normes fondamentales du travail, reconnues au

9. Principes relatifs aux gouvernements d'entreprises de l'OCDE, avril 1999. 
niveau international, sont dorénavant couvertes par les Principes directeurs. On peut toutefois regretter que le texte n'interdise pas explicitement les pires formes de travail des enfants, conformément à la Convention de l'OIT sur ce sujet, adoptée à l'unanimité par la Communauté internationale le 17 juin 1999. Selon la définition de l'OIT, les pires formes de travail des enfants englobent toutes les formes d'esclavage ou pratiques analogues, telles que, par exemple, la vente et la traite des enfants, la servitude pour les dettes et le servage, le travail forcé ou obligatoire ainsi que les travaux susceptibles de nuire à la santé, à la sécurité ou à la moralité des enfants. La Convention de l'OIT exhorte les Etats qui la ratifient à infliger des sanctions pénales à l'encontre de ceux qui se rendent coupables de telles exactions ${ }^{10}$. Il aurait été vivement souhaitable que les Principes directeurs reprennent à leur compte ce combat mondial en faveur du respect de l'intégrité et de la dignité des enfants au travail.

Le chapitre 5 sur l'environnement encourage désormais les multinationales à améliorer leur comportement du point de vue écologique grâce à des mesures telles qu'une meilleure gestion environnementale interne et des règles plus strictes concernant la publication d'informations environnementales. Le texte introduit également le principe de précaution pour l'environnement et la santé humaine. Un projet plus ambitieux - qui présentait la gestion environnementale comme une priorité pour les multinationales, exigeait un rapport régulier sur les performances écologiques et adoptait le principe de la participation publique lors d'actions pouvant avoir un impact sur l'environnement - a toutefois été balayé par l'opposition virulente des représentants de l'économie.

Avec les chapitres 6 et 7, deux nouveaux sujets sont introduits dans les Principes directeurs: la lutte contre la corruption et les intérêts des consommateurs. Ces ajouts ont été salués par les ONG, en particulier celles actives dans ces deux secteurs et qui ont, par leur travail de lobby, grandement contribué à cette adaptation qui n'avait initialement pas été prévue.

\section{FAIBLESSE DE LA MISE EN E EUVRE}

D'une manière globale, ces modifications matérielles ont été accueillies positivement par l'ensemble des ONG ayant participé au processus de révision avec toutefois quelques réserves. Celles-ci auraient en effet souhaité des formulations plus exigeantes à l'encontre des multinationales et ont regretté que les membres de l'OCDE se soient souvent limités aux normes minimales. La principale critique des ONG à l'égard de cette nouvelle version des principes porte cependant sur leur mise en œuvre, qui non seulement n'est pas garantie, mais qui ne fait l'objet d'aucune mesure de contrôle ni d'évaluation indépendante. Cette faiblesse pose le problème de la pertinence et de la crédibilité des recommandations. C'est pourquoi, tout au long des négociations, les ONG ont plaidé en faveur de l'adoption d'un mécanisme de mise en œuvre plus performant ainsi que pour un renforcement des Points de contacts nationaux (PCN), chargés de promouvoir les Principes directeurs dans les pays membres.

Les PCN ont en effet un rôle central. Ils sont responsables de diffuser l'information auprès des multinationales et des milieux d'affaires. Ils fonctionnent égale-

10. «La Convention sur les pires formes de travail des enfants», in «Travail» Le Magazine de l'OIT, $\mathrm{n}^{\circ} 30$, juillet 1999. 
ment comme une agence de renseignement sur toutes les questions liées aux Principes directeurs et à leur application. La nouvelle version des principes a renforcé et précisé leur rôle et leurs responsabilités. C'est en particulier la procédure de résolution de conflits - pouvant surgir sur l'interprétation des Principes directeurs - qui a été clarifiée afin d'assurer un déroulement équitable et neutre pour toutes les parties. Désormais, un PCN peut, par exemple, proposer ses bons offices pour régler un contentieux et est encouragé à cette fin de s'entourer du conseil, non seulement, des milieux économiques, mais aussi des organisations syndicales, des ONG ou d'autres experts. De plus, des réunions et un rapport d'activités annuels ont été prévus afin de permettre une meilleure circulation de l'information.

La faiblesse de ce processus de mise en œuvre vient du fonctionnement très disparate des PNC selon les pays et de l'absence de tout mécanisme international de contrôle et d'évaluation. L'efficacité du système repose presque entièrement sur la bonne volonté des gouvernements de procéder activement à la diffusion des recommandations auprès des multinationales et de gérer de façon transparente et efficace les conflits qui pourraient surgir. La Déclaration de 1976 de l'OCDE, dont les Principes directeurs font partie, n'a pas de force contraignante. C'est un instrument juridique approuvé par le Conseil des ministres de l'OCDE, mais qui ne nécessite pas une ratification de la part des Etats membres. Chaque pays peut donc donner l'importance qu'il veut à son engagement. Jusqu'à aujourd'hui, il n'existe pas d'évaluation détaillée du degré de mise en œuvre. On a cependant toutes les raisons de croire qu'il est très faible. TUAC l'a souligné lors de l'une des premières consultations sur la révision des principes datant de décembre 1997: The Review should focus on the implementation and promotion of the Guidelines which in many Member countries of the OECD is non-existent.

Les PCN sont en principe localisés dans les administrations gouvernementales. En Suisse, par exemple, le Point de contact national est la section «Investissements et droit économique international» auprès du Seco. Jusqu'à aujourd'hui, la diffusion des recommandations auprès des multinationales s'est faite de façon relativement sommaire. Elle consistait principalement en la distribution de la brochure réalisée par l'OCDE à toutes les associations économiques concernées ainsi qu'en l'organisation de quelques conférences. Le groupe de liaison, présenté ci-dessus, a également été l'une des courroies de transmission utilisées par le Seco pour diffuser l'information auprès des multinationales du pays.

Cette situation insatisfaisante en matière de mise en œuvre a poussé les ONG à réclamer l'adoption d'un processus de contrôle neutre, indépendant et ouvert. Elles ont notamment présenté une proposition comportant deux volets. Premièrement, la création d'un mécanisme de souscription volontaire à l'attention des entreprises prêtes à s'engager ouvertement à respecter les Principes directeurs. Deuxièmement, l'adoption d'une procédure de conciliation publique, qui aurait permis à toute partie ou personne concernée de déposer une plainte à l'encontre d'une de ces entreprises ayant publiquement déclaré son engagement. En leur donnant ainsi la possibilité de valoriser leurs efforts auprès de l'opinion publique, ces mesures de mise en œuvre et de contrôle indépendant auraient été un bon moyen pour motiver les multinationales soucieuses d'agir en faveur $\mathrm{du}$ développement durable. Elles auraient aussi renforcé considérablement la crédibilité des Principes directeurs auprès de la société civile et des ONG. 
Mais ces propositions se sont heurtées à de vives oppositions. Le BIAC a estimé qu'un tel mécanisme exigeait la mise en place d'une bureaucratie trop importante et trop chère compte tenu du nombre d'entreprises concernées. De plus, selon lui, les multinationales auraient été peu nombreuses à s'engager publiquement, retirant ainsi aux Principes directeurs leur caractère global. Les sanctions du marché, c'est-à-dire des consommateurs, seraient largement suffisantes pour inciter les multinationales à agir correctement. Quelques pays en développement ont également exprimé des réticences. Le Mexique a même failli retarder l'adoption des nouveaux principes, car ses représentants craignaient que les dispositions environnementales et sociales ainsi que la procédure améliorée de mise en œuvre ne mettent en péril l'attractivité de leur pays pour les investisseurs nord-américains. C'est seulement sous la pression des autres membres, qui ne souhaitaient en aucun cas rouvrir des négociations, et après avoir obtenu toutes les garanties que les Principes directeurs ont bien un caractère non contraignant, que le Mexique a finalement donné son approbation à la révision.

\section{CONCLUSION}

La version révisée des Principes directeurs est une reconnaissance de la part des pays de l'OCDE qu'un nouveau type de partenariat entre gouvernements et multinationales est nécessaire pour amener les entreprises à assumer leur responsabilité dans la promotion du développement durable. La mondialisation de l'économie, caractérisée par la libéralisation des échanges et les dérégulations, a radicalement changé la nature des relations entre les multinationales et les Etats. L'abaissement des frontières a fait émerger une nouvelle priorité qui est celle d'offrir aux acteurs économiques un cadre de fonctionnement avec des droits et des responsabilités globaux et équilibrés correspondant au contexte d'un marché mondial intégré. Réglementer le comportement des multinationales ne peut plus se faire seulement vis-à-vis des Etats, mais de nouvelles préoccupations à caractère universel, sociales et environnementales, doivent désormais être impérativement prises en considération.

Avec l'AMI, l'OCDE a voulu commencer par garantir des droits aux investisseurs sans accorder la même importance aux obligations. Cette stratégie a échoué grâce à la vigilance de la société civile, relayée par les ONG du monde entier. Avec l'adoption des nouveaux Principes directeurs, on est en présence du premier code de conduite multilatéral qui mentionne expressément les normes sociales et environnementales minimales reconnues au niveau international. Ils constituent donc une première référence officielle dans ce domaine, qui pourrait s'avérer particulièrement utile dans le développement d'autres instruments d'incitation tels que, par exemple, les nombreux codes de conduite privés élaborés récemment par les multinationales de façon unilatérale ou en collaboration avec des ONG.

Bien que le caractère non contraignant des principes ne permette pas de garantir véritablement leur efficacité et leur utilité, ils représentent en effet une ébauche d'obligations dans la mesure où les Etats signataires ont accepté de les promouvoir auprès de leurs multinationales. Comme nous l'avons vu ci-dessus, la problématique se situe finalement moins dans le caractère non contraignant des recommandations que dans celui de la mise en œuvre. On peut espérer que le pas réalisé dans la bonne direction sera bientôt suivi par d'autres. Les Etats 
membres de l'OCDE ont en effet décidé de poursuivre les discussions sur l'amélioration du processus de concrétisation. Ils souhaitent notamment concentrer leurs efforts sur la question de la diffusion la plus large possible des Principes directeurs et sur l'intensification des contacts avec les pays non-membres de l'OCDE. L'extension géographique du champ d'application des principes au monde entier pose en effet des problèmes de mise en œuvre supplémentaires, en particulier à cause de l'absence de PCN dans les pays non-membres de l'OCDE, auxquels il s'agit de trouver des solutions adéquates.

Par ailleurs, l'effort de l'OCDE d'agir de façon transparente et ouverte est à saluer. Non seulement les ONG ont pu participer activement au processus de révision, mais elles sont désormais explicitement reconnues comme partenaires dans le cadre des divers mécanismes de consultation prévus au niveau du CIME et des PCN. Au même titre que les milieux économiques et syndicaux, elles peuvent donc nouvellement solliciter des consultations à tout moment au niveau international et national. L'attention que les Principes directeurs continueront à susciter auprès de la société civile constituera donc, sans aucun doute, un facteur stimulant afin que ces derniers deviennent un véritable instrument de promotion pour un comportement responsable des multinationales à l'égard du contexte économique, social et environnemental dans lequel elles évoluent.

\section{SOURCES}

«Les Principes directeurs de l'OCDE dans un monde en voie de mondialisation », Arghyrios A. Fatouros, version révisée d'un document présenté par l'auteur à la Conférence de Budapest sur les Principes directeurs de l'OCDE, novembre 1998, publié par la Direction des affaires financières, fiscales et des entreprises de l'OCDE.

«Mexico digs in over OECD rules», Guy de Jonquières, Financial Times, 23 juin 2000

«OECD Revising Guidelines for Multinationals, Environment, Labor Standards Prominent», Lawrence J. Speer, in International Trade Reporter, vol. 17, No.5, 3 février 2000, p. 193.

\section{INTERNET}

www.oecd.org

Pour le texte intégral des Principes directeurs : www.oecd.org/daf/investment/guidelines/faq.htm 


\title{
3.3. LA RESPONSABILITÉ DES ENTREPRISES DANS LA PERSPECTIVE D'UN DÉVELOPPEMENT DURABLE"
}

\author{
Peter UtTing*
}

\section{INTRODUCTION}

Les institutions de développement et les acteurs qui cherchent à promouvoir un développement durable ${ }^{12}$ ont été rejoints ces dernières années par un autre protagoniste: le grand capital. L'adhésion croissante des chefs d'entreprise à des concepts tels que ceux d'entreprise citoyenne ou de responsabilité sociale de l'entreprise donne à penser que ce milieu commence à reconsidérer ses relations à la fois vis-à-vis de l'environnement et vis-à-vis des nombreux acteurs concernés. Cette nouvelle donne contraste très vivement avec le scénario antérieur dans lequel le grand capital semblait insensible aux préoccupations de certains partenaires et dans une large mesure responsable de la dégradation de l'environnement à l'échelle planétaire.

Dans ce texte, nous verrons dans quelle mesure les grandes entreprises adoptent des politiques et des pratiques qui vont dans le sens d'un développement durable. Après avoir décrit diverses innovations institutionnelles qui sembleraient promouvoir la notion de responsabilité sociale et environnementale des entreprises, nous évaluerons l'impact réel de ces innovations et tenterons de répondre à la question de savoir si le monde des affaires est ou non susceptible d'améliorer sensiblement son bilan environnemental et social.

\section{INNOVATIONS INSTITUTIONNELLES}

A diverses périodes de l'histoire du capitalisme d'entreprise, le monde des affaires a adopté des politiques et des réformes traduisant un certain degré de responsabilité environnementale et sociale. Ainsi, durant les premières décennies du siècle écoulé, de grands industriels tels que Ford et Carnegie non seulement se sont occupés d'œuvres de bienfaisance mais encore ont pris des mesures pour améliorer la situation des travailleurs et des communautés au sein desquelles leurs usines étaient implantées. La notion de responsabilité sociale des entreprises a gagné du terrain après la Seconde Guerre mondiale, avec la démocratie sociale et l'adoption de la législation sur la protection sociale. Cette évolution a été beaucoup moins marquée dans la plupart des pays en développement, encore que certaines entreprises semi-publiques d'Afrique et de grandes

* Peter Utting: coordinateur de projets à l'Institut de recherche des Nations Unies pour le développement social (UNRISD), Genève.

11. Le présent texte est une version abrégée d'un article préparé pour l'UNRISD sous le titre Business Responsibility for Sustainable Development (Utting, 2000a).

12. Selon la définition de la Commission Brundtland, le développement durable est un «développement qui répond aux besoins des générations actuelles sans compromettre celui des générations futures à satisfaire leurs propres besoins » (WCED, 1987). Il s'agit là bien sûr d'une tâche difficile, le développement devant être considéré plutôt comme un processus que comme un objectif. On pourrait aussi formuler autrement les choses en disant qu'il s'agit d'un processus qui réduit au maximum les contradictions entre croissance économique, protection de l'environnement et bien-être social (Utting, 1993). 
entreprises familiales d'Asie de l'Est aient élaboré des politiques sociales dignes d'être notées.

La notion contemporaine de responsabilité des entreprises est à maints égards différente. Elle a cours dans un beaucoup plus grand nombre de pays, les forces qui la gouvernent se sont diversifiées, les orientations politiques et les accords institutionnels sur lesquels elle s'appuie sont différents et - récemment encore - l'accent était davantage mis sur l'environnement que sur les aspects sociaux.

Le Sommet de la Terre de juin 1992 a constitué à cet égard un événement déterminant. Cette conférence a attiré l'attention du grand public et des entreprises sur la question de la dégradation de l'environnement et du développement durable. Le Programme Action 21, adopté à cette occasion, contient 32 dispositions concernant les activités des sociétés transnationales (STN) (UNCTAD, 1996 : 3). Il enjoint les gouvernements du monde entier, les entreprises, les institutions internationales de développement et les organisations non gouvernementales (ONG) de collaborer en vue de rendre plus compatibles croissance économique et protection de l'environnement. L'attitude conflictuelle des décennies précédentes, dans laquelle on a vu les organisations et les groupements partisans de la réglementation et de la redistribution s'opposer avec véhémence aux STN, a perdu de sa vigueur. A une réglementation émanant des pouvoirs publics, on préfère aujourd'hui l' «auto-réglementation des entreprises » et les initiatives volontaires, considérées comme les meilleurs moyens de favoriser l'adoption d'instruments et de méthodes reflétant la responsabilité environnementale des entreprises. Ce sont, par exemple, les codes de conduite, les technologies propres, l'analyse du cycle de vie des produits ${ }^{13}$, les rapports sur l'environnement et la certification environnementale.

D'importants changements institutionnels ont eu lieu dans les années 90. La Déclaration de Rio invitait le monde des affaires à adhérer au «principe de précaution ${ }^{14}$, à prendre des mesures destinées à promouvoir la responsabilité environnementale et à encourager la mise au point et la diffusion de technologies respectueuses de l'environnement (Annan, 1999). Sous l'égide d'un petit groupe de très grandes STN, une importante partie du monde des affaires a explicitement reconnu que les entreprises devaient faire le ménage chez elles et agir plus énergiquement en faveur de la protection de l'environnement et du développement durable. Une association de STN, le Conseil des entreprises pour un développement durable (BCSD), a exercé une influence particulièrement importante sur le processus de la Conférence des Nations Unies sur l'environnement et le développement. A travers des publications telles que Changing Course (Schmidheiny, 1992), elle a préconisé une remise à plat de la stratégie des entreprises en matière d'exploitation et de gestion des ressources naturelles et prôné la solution dite de l'«éco-efficacité». La Chambre de commerce internationale (CCI), qui, en 1991 avait énoncé 16 principes écologiques dans la Charte des entreprises pour un développement durable, s'est également fait

13. L'analyse du cycle de vie porte sur les répercussions écologiques de tel produit ou service à tous les stades de production, de commercialisation et d'élimination.

14. Selon ce principe, il convient de prendre des mesures de protection de l'environnement même dans les cas où les causes et les effets de la dégradation de l'environnement ne sont pas formellement démontrés d'un point de vue scientifique. 
entendre à Rio en affirmant que le monde des affaires s'était désormais engagé sur la voie du développement durable (Schmidheiny et al., 1997).

A la suite du Sommet de la Terre, la promotion d'une gestion écologique des entreprises s'est intensifiée avec la création de nombreuses associations d'entreprises - baptisées «réseaux d'entreprises vertes» - aux objectifs explicitement écologiques. Vers la fin des années 90 , certaines de ces associations procédèrent à des regroupements dont le plus important fut la fusion, en 1995, du BCSD et du Conseil mondial des entreprises pour l'environnement (WICE) qui aboutit à la création du Conseil mondial des entreprises pour un développement durable (WBCSD) (Hansen, 1999a). L'année suivante, l'Organisation internationale de normalisation (ISO) adopta une série de normes génériques régissant les systèmes de management environnemental des entreprises. Ces normes, appelées ISO 14000, sont de plus en plus utilisées comme des points de référence et des critères de certification pour faciliter le commerce international (UNCTAD, 1996).

En outre, de nouvelles institutions régionales influent elles aussi sur la politique et la pratique des entreprises. Par exemple, le système communautaire de management environnemental et d'audit (EMAS), a été institué en 1993 pour favoriser une bonne gestion de l'environnement dans l'industrie manufacturière et comporte des normes plus rigoureuses que l'ISO 14001. La Commission de la coopération environnementale de l'ALENA (Accord de libre-échange nord-américain) supervise les questions d'environnement au Canada, aux Etats-Unis et au Mexique. Le Conseil centraméricain du développement durable, institué en 1989 par les présidents des pays de la région, encourage activement la planification d'un développement durable et les projets correspondants dans sept pays et dans des branches d'activité telles que l'exploitation forestière et le tourisme, dans lesquelles les milieux industriels et commerciaux ont une forte participation.

Beaucoup d'associations sectorielles et d'entreprises isolées sont favorables à ce que l'on appelle les «initiatives volontaires» qui incitent le milieu des affaires à pratiquer 1'autoréglementation d'entreprise ou à coopérer avec les pouvoirs publics à la négociation et à l'application de règles mutuellement acceptées. Parmi les initiatives volontaires les plus importantes, figurent divers codes de conduite ou principes directeurs établis par des associations professionnelles nationales et internationales. Il convient de mentionner dans ce contexte les nombreux programmes de «gestion responsable» des associations nationales de l'industrie chimique, qui obligent les entreprises membres à améliorer constamment leurs résultats dans les domaines de la santé, de la sécurité et de l'environnement (ILO, 1999) ${ }^{15}$ et la Charte mondiale de l'environnement de Keidanren, adoptée en 1991, qui engage les entreprises du Japon à œuvrer en faveur d'un développement humain, écologique et économique sain (UNCTAD, 1996 : 148). En 1999, la charte de la CCI, dont il est question plus haut, avait été adoptée par 2300 entreprises - soit près d'un tiers des membres de cet organisme. ${ }^{16}$

Pendant la seconde partie des années 90, le grand capital s'est de plus en plus intéressé à la responsabilité sociale des entreprises. En 1995, l'Association des entreprises pour le Sommet mondial sur le développement social (BUSCO) a

15. En 1996, environ 40 associations nationales de fabricants de produits chimiques, dont les membres représentent $86 \%$ de la production mondiale, avaient adopté le programme de gestion responsable.

16. Communication personnelle avec un responsable de la CCI, 17.8.1999. 
présenté à la conférence de Copenhague une déclaration de principes et un programme d'action en 16 points que les entreprises étaient incitées à adopter afin d'accroître leur contribution au progrès social (BUSCO, 1995). Des organisations telles que le WBCSD ont elles aussi diversifié leurs activités en s'occupant plus directement des questions liées à la responsabilité sociale des entreprises (Watts et Holmes, 1999). En 1997, des organismes internationaux tels que la Fédération mondiale de l'industrie d'articles de sports et le Conseil international des industries du jouet ont adopté des codes portant en particulier sur les conditions de travail et le travail des enfants (Wild, 1998).

Ces dernières années, certains organismes du secteur privé ont inclus dans leurs codes ou principes directeurs des engagements concernant les droits de l'homme. En 1997, par exemple, Shell a révisé sa déclaration de principes généraux sur la conduite des affaires pour marquer son adhésion à la Déclaration universelle des droits de l'homme (UNCTAD, 1999). Le monde des entreprises a également participé à la formulation de la norme internationale, «Social Accountability (SA) 8000 », élaborée en 1998 par le Council on Economic Priorities Accreditation Agency (CEPAA) ${ }^{17}$. Incorporant plusieurs normes de l'OIT et de l'ONU relatives aux conditions de travail et aux droits de l'homme, SA 8000 vise à améliorer les conditions de vie et de travail aussi bien dans les pays en développement que dans les pays industrialisés (Wild, 1998).

L'intérêt pour les questions environnementales et sociales est également manifeste dans un autre grand programme international, lancé à la fin des années 90: la Global Reporting Initiative (GRI). Il s'agit d'une action collective visant à mettre sur pied un système commun de notification sur l'impact économique, écologique et social des entreprises ${ }^{18}$. En 1999, 21 sociétés ont testé les lignes directrices du programme GRI dans leur version préliminaire.

Le changement d'attitude des entreprises se reflète aussi dans le phénomène des «partenariats». La décennie écoulée à été une période de rapprochement considérable entre le monde des affaires et certains acteurs dont les gouvernements, les institutions des Nations Unies et certaines ONG, qui s'efforçaient auparavant d'exercer une influence sur les entreprises par des directives, des codes de conduite internationaux et une politique d'affrontement. De nos jours, ces acteurs adoptent plutôt une attitude de collaboration avec le monde des affaires.

Certaines des plus grandes entreprises mondiales veulent établir des relations de partenariat avec les ONG et les institutions des Nations Unies. Il ressort d'une analyse de l'état des entreprises vertes réalisée en 1998 que, pour les entreprises clairvoyantes «le partenariat s'est rapidement imposé comme la stratégie du choix » (Frankel, 1999 : 11). Certains partenariats conclus ces dernières années ont été entourés de beaucoup de publicité. Ainsi, en 1996, le WWF-International a lancé un partenariat avec la société Unilever, qui est le plus grand acheteur mondial de poisson congelé, en vue d'instituer au sein de l'industrie des fruits de mer des incitations économiques pour une «pêche viable» à l'échelle mondiale. Ces efforts ont abouti à la création du Conseil d'intendance des mers

17. Le CEPAA a changé de nom en 2000; il s'appelle désormais Social Accountability International

18. La GRI a été créée en 1997 par la Coalition for Environmentally Responsible Economies (CERES) et le Programme des Nations Unies pour l'environnement (PNUE). Elle est censée devenir un organisme international indépendant en 2002. 
(MSC). La Fairtrade Foundation - coalition d'organisations de développement international, d'associations de consommateurs et d'organisations militant pour un commerce équitable - a lancé un projet de collaboration avec des entreprises britanniques en vue d'élaborer des directives pratiques régissant les relations avec les fournisseurs du Sud. En 1998, la British Petroleum s'est alliée au Environmental Defense Fund et General Motors à l'Institut des ressources mondiales. A la fin de 1998, 17 entreprises de Fortune 500 finançaient le Pew Center on Global Climate Change (Frankel, 1999).

Si dans le passé la collaboration entre les entreprises et les ONG était souvent axée sur la bienfaisance, aujourd'hui, beaucoup d'ONG interviennent auprès des entreprises sur des questions de fonctionnement interne (Murphy et Bendell, 1999) - par exemple, en les aidant à élaborer des codes de conduite, à mettre au point des systèmes de rapports sur l'environnement ou à réaliser des audits sociaux indépendants. Ces relations entre les ONG et les entreprises relèvent de ce que l'on appelle la «réglementation civile» - les entreprises étant poussées à se conformer non seulement à leurs propres règles (autoréglementation d'entreprise) ou à celles du gouvernement (conformité juridique), mais également aux normes et aux règles dans une certaine mesure définies par les institutions de la société civile (Murphy et Bendell, 1999).

Le nombre de partenariats auxquels participent des entreprises et des institutions des Nations Unies telles que la CNUCED, le PNUE, l'ONUDI et l'OMS, a lui aussi fortement augmenté ces dernières années (Tesner, 1999; Utting, 2000b). En 1999, la CCI s'est ralliée à l'appel lancé par le secrétaire général des Nations Unies, Kofi Annan, en faveur d'un Pacte mondial entre les Nations Unies et le monde des affaires, dans le cadre duquel les entreprises adhéreraient volontairement aux normes des Nations Unies qui portent sur la protection de l'environnement, les conditions de travail et les droits de l'homme, et collaboreraient à cette fin avec les institutions concernées. Ce Pacte mondial, qui a été officiellement proclamé en juillet 2000, vise à obtenir l'appui de 100 grandes sociétés et de 1000 entreprises en trois ans.

\section{UN CHANGEMENT SIGNIFICATIF?}

Toute cette activité favorise-t-elle le développement durable dans le Sud? Les nombreuses initiatives prises par le monde des affaires suffisent-elles pour "combattre les ravages de l'inertie ambiante et créer une dynamique propice à un développement durable?» (Schmidheiny, 1992). Les avis sur cette question sont en général très tranchés.

Tout en admettant que le changement ne se produit pas encore partout au même rythme, le WBCSD (Schmidheiny et al., 1997) constate une évolution de la politique et de la pratique des entreprises, qui est à ses yeux révélatrice d'un changement d'optique, à savoir:

- passage d'une concentration sur le coût et les difficultés engendrés par l'application du concept de développement durable à la reconnaissance des économies et des avantages qui peuvent en découler;

- passage de la lutte contre la pollution en fin de cycle à l'utilisation de technologies propres et performantes à tous les stades de la production et à l'idée que développement durable et développement de l'entreprise ne font qu'un; 
- passage d'une pensée linéaire, ne tenant compte que du volume d'activité, à une approche systémique et au recyclage;

- acceptation de l'idée que les questions écologiques et sociales ne relèvent pas exclusivement de la compétence des services techniques ou des experts mais sont l'affaire de l'entreprise dans son ensemble;

- abandon du postulat de la confidentialité au profit du principe d'ouverture et de transparence;

- abandon des manœuvres de couloir au profit d'un dialogue plus ouvert avec toutes les parties prenantes.

Dans cette nouvelle optique, un nombre croissant d'entreprises non seulement adoptent des mesures novatrices reflétant leur responsabilité environnementale et sociale, mais encore remplacent leur approche étroite, au coup par coup et technique par une démarche holiste, systémique et ouverte aux intérêts de partenaires multiples.

Cet avis est contredit par ceux qui accusent les entreprises de jeter de la poudre aux yeux ou de "verdir» leur image. Selon Corporate Watch, "des entreprises foncièrement dévastatrices sur les plans écologique et social essaient de préserver et de développer leurs marchés en se faisant passer pour des amies de l'environnement et des chefs de file de la lutte contre la pauvreté » (Corporate Watch, 1996). Dans ces conditions, beaucoup de politiques et d'initiatives d'entreprise sont inconsistantes, ne sont pas appliquées ou reviennent à n'apporter que des retouches de pure forme à un système qui incite au maintien du statu quo (Welford, 1997).

Concrètement, il est extrêmement difficile d'évaluer le degré actuel de responsabilisation environnementale et sociale des entreprises. C'est un fait notoire que les «données» fournies dans la plupart des textes écrits sur ce sujet proviennent d'anecdotes, d'un nombre infime d'études de cas ou encore de vastes généralisations sur le comportement des entreprises dans le contexte du capitalisme et de la mondialisation. Le tableau se brouille encore plus lorsque l'on constate que beaucoup d'entreprises citées en exemple pour leurs «bonnes pratiques» sont par ailleurs désignées comme faisant partie des mauvais élèves (voir encadré ci-dessous). Les enquêtes visant à recenser les entreprises qui ont amélioré leur performance environnementale et sociale sont relativement rares et encore mesurent-elles généralement les changements de procédure et de politique plutôt que l'impact environnemental et social de celles-ci.

La plupart des observateurs s'accordent à dire que, dans l'état actuel des choses, la responsabilisation des entreprises est pour le moins fragmentée et inégale. Très peu d'entreprises ont montré la voie à suivre dans ce domaine. Au début de la décennie, Hawken écrivait que «approximativement 2000 entreprises motivées ont réalisé ensemble un chiffre d'affaires d'environ 2 milliards de dollars, soit un centième de $1 \%$ des 20 billions de dollars engrangés par les 80 à 100 millions d'entreprises qui existent dans le monde» (Hawken, 1993, cité dans Thomson, 1998). Depuis que cette estimation a été faite, certaines des plus grandes sociétés mondiales ont adhéré au mouvement, mais elles ne constituent qu'une faible proportion des 63'000 STN du monde (UNCTAD, 2000), et dans la plupart des cas, seule une petite partie de leurs activités est concernée. 


\section{Box: Best or Bad Practice?}

Asea Brown Boveri $(A B B)$, the Swedish/Swiss engineering corporation, is an active member of the WBCSD. It is "a world leader in developing eco-efficient technologies in a wide range of industry areas from electricity transmission to transportation, and is building a global network of joint ventures and strategic alliances to install these technologies in many developing and transition economies" (Nelson, 1996 : 163). ABB has also "faced sustained campaigns by environmentalists and human rights advocates against its involvement in various hydro projects, including the Three Gorges Project in China and the now indefinitely postponed Bakun Dam in Malaysia" (TRAC, 1999 : 1).

Aracruz Celulose, the world's largest exporter of bleached-eucalyptus pulp, is often cited for its efforts to promote sustainable development through its tree planting, harvesting and pulp production processes in Brazil (Sargent and Bass, 1992). "Since its establishment, the company has earned a positive reputation both nationally and internationally for its efforts to incorporate social and environmental factors into its corporate vision" (Nelson, 1996 : 202). Certain investigations, however, have revealed a very different picture: "... its eucalyptus trees have dried streams, destroyed the local fauna, impoverished the soil, impeded the regrowth of native plant species, and drastically reduced the area available for cultivating basic foodstuffs ... This is not to mention land concentration and the expulsion of the rural population ..."(Goncalves et al., 1994, quoted in Carrere, 1999)

Dow Chemical is a US corporation which was selected to participate in one UN-business partnership for, inter alia, "abid[ing] by the highest standards of human rights, environmental and labor standards and norms, as defined by UN agencies" (UNDP, 1999a). According to the Transnational Resource and Action Center (TRAC) "... Dow Chemical is probably the world's largest root source of dioxin - a chlorine by-product closely associated with reproductive disorders, birth defects, increased rates of cancer, and endocrine disruption. ... Dow has regularly exported pesticides unregistered in the U.S. for use in developing countries." (TRAC, 1999)

General Motors (GM), the world's largest automobile manufacturer, is involved in various environmental protection initiatives and partnerships. It is a Charter Partner to the Climate Wise Program, promoted by the US government to encourage energy efficiency (WBCSD, 1999 : 13), and in 1998 entered into a partnership with the World Resources Institute to "define a long-term vision for protecting the earth's climate and the technologies and policies for getting there" (WRI, 1998). Simultaneously, however, GM "maintained its membership in the hardline [Global Climate Coalition] ... [which] continued to be a bastion of reaction and misinformation ...., and the Business Roundtable, which opposes the Kyoto Protocol ..." (Frankel, 1999 : 11).

Mitsubishi Group, has been actively cultivating an image of environmental responsibility through advertising and specific environmental projects. At the same time, it is reputed to be "a leading destroyer of tropical (and non-tropical) forests“ (Greer and Bruno, 1996 : 182). In spring 1998, Mitsubishi was a recipient of the Corporate Watch award for companies excelling in greenwash. In Mexico, the corporation has come under fire for its plans to vastly expand salt production in Baja California, which would have potentially serious implications for a local biosphere reserve, fishing communities and conflicts over land tenure and scarce natural resources such as water (Barkin, 1999).

Novartis, the Swiss life science corporation, is another member of the WBCSD. It is often cited for its efforts in the fields of poverty alleviation and environmental protection. In 1992, for example, the company established a five-year programme to reduce the environmental and social impacts of pesticide use in the Dominican Republic (Watts and Holme, 1999 : 16), and the Novartis Foundation has been active in the field of corporate philanthropy (Novartis, 1998). Despite company claims to be committed to "sustainable development", however, there are concerns that the fundamental Precautionary Principle, which business was asked to uphold at the Earth Summit in 1992, is being ignored by virtue of the company's promotion of genetically modified crops.

Rio Tinto, the British mining company, is often cited for its standards of environmental reporting and for promoting "continued social development" and "sustainable livelihood" in areas such as East Kalimantan, where the company's large mines approach closure (Watts and Holme, 1999 : 10). Yet, according to TRAC, the corporation "has created so many environmental, human rights, and development problems that a global network of trade unions, indigenous peoples, church groups, and community activists has emerged to fight its... [alleged] complicity in, or direct violations ... in Indonesia, Papua New Guinea, Philippines, Namibia, Madagascar, the United States and Australia ..." (TRAC, 1999 : 1) 
L'association d'entreprises la plus importante à cet égard est peut-être le WBCSD, qui ne compte pourtant que 120 membres, même si quelques-unes des plus grandes STN y sont représentées. L'impression d'un changement plus profond provient en partie du fait que les STN et les associations commerciales ou industrielles concernées sont des acteurs de poids sur la scène internationale et n'épargnent aucun effort pour faire connaître leur nouvelle politique par le truchement des médias, de la publicité d'entreprise, de publications, de conférences et des institutions internationales. Elle découle aussi en partie de l'abondante littérature publiée sur les «bonnes pratiques» et sur le noyau relativement restreint d'entreprises qui ont pris la tête du mouvement. Ce type d'information est quelquefois interprétée à tort comme reflétant l'attitude d'une beaucoup plus vaste galaxie. $^{19}$

Dans les pages qui suivent, nous démontrerons la faible ampleur du changement en insistant plus particulièrement sur la situation des pays en développement. Ce changement se caractérise par des réformes très fragmentaires des politiques et méthodes des entreprises ainsi que par un écart d'envergure entre discours et réalité alors que le modèle de croissance économique dominant continue d'encourager des pratiques qui portent atteinte à l'environnement et passent outre les préoccupations de divers acteurs concernés (Utting, à paraître).

\section{$\square$ Progrès embryonnaire et au coup par coup}

Les initiatives des entreprises prennent surtout la forme de codes de conduite ou de déclarations de principe sur des questions environnementales ou sociales. Comme nous l'avons vu plus haut, un nombre croissant d'entreprises et d'associations sectorielles élaborent des codes de conduite et des principes d'action.

Malgré la vague d'enthousiasme que suscitent actuellement les codes dans le monde, la proportion d'entreprises qui en adoptent demeure relativement faible dans la plupart des pays. Ainsi, l'OCDE a récemment inventorié les entreprises dotées de code et n'en a trouvé que 233 (OECD, 1999a). Même dans les pays en développement, où l'on pourrait s'attendre à de meilleurs résultats, la situation n'est pas particulièrement encourageante. Par exemple, au Costa Rica - pays internationalement reconnu pour ses mesures de protection de l'environnement - un tiers des grandes entreprises ont une politique de l'environnement (Pratt et Fintel, 1999). En outre, les codes ont en général une portée très limitée. Il ressort par exemple d'une étude des sociétés canadiennes que «la majorité des grandes entreprises qui ont des activités ou s'approvisionnent à l'étranger ne mentionnent même pas les droits les plus élémentaires de la personne humaine» (Forcese, 1996). Les sujets traités en priorité dans les codes sont en général ceux auxquels les consommateurs sont sensibles - travail des enfants, discrimination, protection de l'environnement, etc. - et non ceux relevés par des organisations internationales telles que l'OIT. Ainsi, «la liberté syndicale et le droit de négociation collective sont presque toujours absents » (Wild, $1998: 32$ ).

Si les codes sont loin d'être chose courante, leur application laisse encore plus à désirer. En général, ils ne contiennent que des principes vagues et des déclarations d'intention, qui ne sont pas effectivement mis en œuvre (Kolk et al.,

19. Une étude menée auprès des filiales de STN dans trois pays d'Asie, qui a obtenu un faible taux de réponse, met en garde contre une telle erreur d'interprétation (Hansen, 1999b). 
1999). A la faveur d'une étude des lignes directrices élaborées par 26 associations industrielles internationales à l'intention de leurs membres, la CNUCED a constaté que «la plupart ne demandaient pas aux signataires de s'engager à respecter les principes énoncés ni à prendre les mesures recommandées et que très peu exigeaient de leurs membres une quelconque discipline» (UNCTAD, 1996 : 7). Bien souvent, les salariés et les consommateurs ignorent l'existence de ces codes et les entreprises ne précisent pas la nature des sanctions applicables en cas de non-observation des règles qu'ils contiennent. L'absence quasi générale d'auto-évaluations dignes de ce nom ou de vérifications indépendantes du respect des codes est particulièrement préoccupante (Dommen, 1999; ILO, 1999; UNCTAD, 1996). Selon l'étude canadienne susmentionnée, «la plupart des codes ne prévoient aucun contrôle indépendant. Les entreprises semblent réticentes à l'idée de rendre leurs codes publics même lorsqu'elles disent y avoir inclus des dispositions relatives aux droits de l'homme. Un tel manque d'empressement va à l'encontre de la transparence préconisée par les experts, aux stades de l'élaboration, de l'application et du suivi des codes» (Forcese, 1996).

Un autre domaine dans lequel des progrès sont manifestes mais de faible ampleur est l'établissement de rapports sur l'environnement et les questions sociales. Il ressort d'une étude de 100 «entreprises pionnières», réalisée en 1994 par le PNUE, que les deux tiers de ces entreprises (64\%) ne publiaient en guise de rapports que des brochures ou des comptes-rendus annuels contenant plus de texte que de chiffres. Seulement $5 \%$ contenaient des données valables sur les résultats obtenus et aucun ne pouvait être assimilé à un «rapport sur le développement durable $»^{20}$. «Quelle que soit la dénomination des rapports et quel que soit le nombre d'apparitions de l'expression "développement durable" dans le texte, l'action des entreprises dans ce domaine est encore très timide» (UNEP, 1994 : 67). Une étude complémentaire réalisée en 1997, qui portait également sur une centaine d'entreprises, fait état de progrès notables, la plupart de ces entreprises fournissant des données utiles sur certains points (UNEP and Sustainability, 1997). Les auteurs constatent cependant «une réticence à évaluer les résultats obtenus en fonction d'indicateurs de durabilité ». Les rapports d'une seule entreprise contenaient la quasi-totalité des informations requises.

Lorsque la présentation de rapports est chose admise, ceux-ci contiennent souvent une information très sélective. Selon Zadek, seules quelques entreprises telles que The Body Shop et Traidcraft au Royaume-Uni ou encore Ben \& Jerry aux Etats-Unis - ont recours à des «méthodes systématiques, globales, rigoureuses et soumises à une vérification externe pour examiner, divulguer et améliorer leur bilan social» (cité dans Nelson, 1996 : 86). Des initiatives telles que la GRI, dont il a été question plus haut, visent à accroître la comparabilité, la rigueur et la crédibilité des données mais elles ne sont encore que dans leur phase de démarrage. A la fin de l'an 2000, seulement 26 entreprises avaient, de source sûre, adopté les directives de la GRI.

20. Les rapports sur le développement durable «doivent être établis sur la base d'un recours extensif aux méthodes quantitatives (analyse du cycle de vie et bilans de matière) et d'une comparaison étroite avec les rapports nationaux et sectoriels sur le développement durable, conformément à des objectifs convenus à l'avance» (UNEP, 1994 :8). 
Rares sont les pays en développement dans lesquels il existe des mesures d'incitation ou de pression poussant les entreprises à rendre compte de leurs politiques environnementale et sociale. Selon une étude, «produire des rapports coûte du temps et de l'argent et exige des compétences, si bien que beaucoup d'entreprises de pays en développement ne seraient disposées à le faire que s'il existait des règlements à cet effet et si ces règlements étaient strictement appliqués. Ainsi, l'absence de tels règlements, entre autres, donne à penser que les rapports sur la politique environnementale et sociale continueront à rebuter les entreprises des pays en développement, du moins dans l'avenir proche» (Shiraz, 1998).

Progrès embryonnaires aussi en ce qui concerne la certification environnementale. Deux grandes mesures prises dans ce domaine sont la mise en œuvre des systèmes de certification de la norme ISO 14001 et du Forest Stewardship Council (FSC). A la fin de 1999, 14'106 certificats de conformité à la norme ISO 14001 avaient été délivrés dans le monde, chiffre qui paraît bien insignifiant comparé au nombre potentiel d'entreprises et de sites de production qui devraient adhérer à ce système. A elles seules, les filiales de STN étaient estimées à 690'000 à la fin des années 90 (UNCTAD, 2000). Bien que le nombre de certificats ISO sur le management environnemental délivré chaque année augmente, seulement 6219 ont été attribués en 1999. Cela est très peu comparé aux 72’000 certificats délivrés en 1999 dans la série ISO 9000 qui porte sur le management de la qualité (ISO, 2000).

Dans son volumineux rapport de 1996 sur l'industrie mondiale de la pâte à papier, l'Institut international pour l'environnement et le développement (IIED) indiquait que près de 6 millions d'hectares de forêts avaient fait l'objet d'une certification, ce qui représentait à peine $0,5 \%$ du commerce mondial (IIED, 1996 : 62). Au début de 1999, la superficie certifiée par des organismes accrédités par le FSC était passée à 15 millions d'hectares (dont un quart dans les pays en développement), mais cela représente encore moins de $1 \%$ des forêts mondiales hors zones protégées ${ }^{21}$. Même au Costa Rica où les exploitants forestiers ont adhéré au principe de la promotion d'une exploitation écologiquement viable des forêts par le biais de la certification, seulement 25'000 hectares, gérés par sept institutions, ont été certifiés (FSC, 1999). Cela représente $5 \%$ du demi-million environ d'hectares de forêts hors zones protégées ${ }^{22}$.

\section{$\square$ Discours et réalité}

Lorsqu'elles évoquent leur responsabilité sociale et environnementale, les entreprises laissent souvent entendre qu'elles ont accompli des progrès impressionnants en très peu de temps. Pourtant, leur discours est loin de concorder avec la réalité. En général, leurs affirmations surpassent de loin leur bilan réel. Une étude réalisée en Amérique latine a, par exemple, fait la lumière sur deux expériences qualifiées de «réussites» dans les publications qui traitent de la responsabilité des entreprises - la prospection de la biodiversité au Costa Rica par la gigantesque firme américaine de produits pharmaceutiques, Merck \& Co., et les

21. D'après les données de la FSC (1999) et les estimations des zones forestières et des zones protégées fournies pour 1996 dans World Development Indicators, 1999 (Banque mondiale, 1999).

22. D'après les estimations des zones forestières et des zones protégées fournies pour 1996 dans World Development Indicators, 1999 (Banque mondiale, 1999). 
activités brésiliennes du fabricant de pâte à papier, Aracruz Cellulose ${ }^{23}$. Ce travail a révélé, dans le cas de Merck \& Co., une exagération des prétentions par rapport aux résultats obtenus et, dans le cas d'Aracruz, des exemples d'irresponsabilité sur les plans environnemental et social.

En Amérique centrale, une évaluation indépendante a récemment dévoilé d'autres cas d'affirmations exagérées ou partisanes dans deux des branches d'activité - l'industrie chimique et la sylviculture - le plus souvent citées pour leurs programmes de management environnemental. Evaluant un projet autour duquel l'industrie internationale des pesticides a fait beaucoup de publicité au Guatemala ${ }^{24}$, l'Union internationale des travailleurs de l'alimentation et de l'agriculture (UITA) a découvert que si les indicateurs de performance choisis étaient en apparence excellents, la conception et l'exécution du projet, elles, laissaient gravement à désirer. Certes, 330'000 agriculteurs, ménagères, étudiants et autres avaient bénéficié d'une formation sur l'emploi des pesticides de 1991 à 1994, mais les méthodes de formation étaient défaillantes. Aucun suivi intensif à long terme n'a été prévu, la question des technologies appropriées dont la lutte intégrée contre les parasites - n'a pas été traitée et les techniques de formation participative ont été écartées. Qui plus est, les salariés agricoles, c'est-à-dire la majorité des utilisateurs de pesticides, étaient exclus du projet, ce qui contredit sérieusement l'affirmation de l'industrie des pesticides selon laquelle elle entendait favoriser la bonne gestion des produits ${ }^{25}$, du stade de la livraison à celui de l'utilisation. Etant donné que la formation s'adressait principalement aux agriculteurs clients des fabricants de pesticides et a passé sous silence les méthodes alternatives de lutte phytosanitaire, l'industrie peut être soupçonnée d'avoir en réalité conçu ce projet pour des raisons de stratégie commerciale (Hurst, 1999). Les problèmes mis à jour par ce type d'enquête mettent en évidence l'importance de l'évaluation indépendante et la nécessité d'inclure une vérification externe dans les initiatives privées des entreprises.

Les exploitants forestiers d'Amérique centrale semblent adhérer au principe de la vérification indépendante mais des doutes subsistent quant à la manière de procéder. Aux critiques de certains écologistes, selon lesquelles la prétendue «exploitation écologiquement durable des forêts » risque tout de même de causer de graves dommages écologiques (Colchester, 1990; World Rainforest Movement, 1999), sont venues s'ajouter d'autres inquiétudes. Par exemple, une étude portant sur une plantation de teck certifiée du Costa Rica, a révélé que le mode de fonctionnement de cette entreprise n'était pas conforme à plusieurs principes et critères du FSC. Elle relève par exemple l'utilisation constante de pesticides extrêmement toxiques qui sont interdits dans de nombreux pays et les conditions

23. Cf. Silvia Rodriguez et Mara Antonieta Camacho, «Bioprospecting in Costa Rica: Facing New Dimensions of Social and Environmental Responsibility» (1999) et Ricardo Carrere, «The Environmental and Social Effects of Corporate Environmentalism in the Brazilian Market Pulp Industry » (1999).

24. L'industrie internationale des pesticides, secouée par la catastrophe de Bhopal (Inde) en 1984 et ses retombées, a pris plusieurs mesures importantes dans le cadre de son programme Responsable Care et des projets Safe Use au Guatemala, au Kenya et en Thaillande.

25. La notion de bonne gestion des produits renvoie à l'obligation que les industriels ont d'assumer la responsabilité des déchets et dommages écologiques provenant d'un produit pendant tout le cycle de vie de celui-ci, soit de l'extraction ou du traitement des matières premières à leur élimination et à leur recyclage en passant par la fabrication et la commercialisation (Welford et Jones, 1996 :252). 
dangereuses dans lesquelles ces produits sont utilisés par un personnel qui n'a pas reçu la formation nécessaire et n'est pas équipé de vêtements de protection. Les auteurs de cette étude font en outre remarquer que des ONG internationales de défense de la nature bien connues cautionnent de fausses affirmations concernant les méthodes de gestion et la performance environnementale et économique de l'entreprise (Romeijn, 1999). Ils démontrent que mieux vaut ne pas considérer comme allant de soi l'autonomie de ceux qui effectuent les contrôles, la rigueur de leurs méthodes et le sérieux de leurs critères ou objectifs.

Autre grande critique de certaines formes de certification: l'évaluation porte sur le management environnemental et non sur l'impact écologique ${ }^{26}$ : Votre entreprise a-t-elle une politique de l'environnement? et non pas «dans quelle mesure votre entreprise a-t-elle réduit ses émissions ou le volume d'énergie qu'elle utilise ?» Le rapport entre l'amélioration du management environnemental et l'impact n'est pas toujours aussi évident qu'on pourrait le penser. Comme le souligne une étude portant sur des multinationales du Nord implantées aux Etats-Unis, ce rapport est parfois «curieusement faible» (Levy, 1995 : 57). Les grandes entreprises, notamment, se sont avérées être beaucoup plus solides sur le plan des orientations déclarées que sur celui des résultats effectifs ${ }^{27}$.

En ce qui concerne les aspects sociaux, les entreprises proclament souvent qu'elles anticipent les problèmes et améliorent sensiblement la situation des travailleurs alors qu'en réalité, les réformes sont insignifiantes. Par exemple, la campagne «Vêtements propres » émet des doutes certains sur le fait que Nike et Reebok auraient, comme ils le prétendent, augmenté de 40\% en 1998-1999 les salaires des ouvriers qui fabriquent des chaussures de sport en Indonésie: "présentée hors contexte comme cela a été le cas, cette augmentation peut paraître énorme. Mais le tableau change du tout au tout lorsqu'on la compare aux difficultés causées par l'inflation de 70\% qui a sévi en 1997-1998, la dévaluation massive et les profits qu'en ont tirés des entreprises telles que Nike et Reebok. Certes, l'augmentation en question est largement supérieure aux $15 \%$ rajoutés par le gouvernement au salaire minimum, mais il n'en demeure pas moins que les salaires payés par Nike et Reebok n'assurent toujours pas des conditions d'existence convenables » (Campagne «Vêtements propres », 1999).

Cependant, les affirmations les plus excessives ne sont pas celles qui portent sur telle ou telle mesure de protection environnementale ou sociale mais celles qui propagent l'idée que les entreprises œuvrent en faveur du «développement durable». Beaucoup de sociétés, de fondations privées et d'associations ou de réseaux d'entreprises collent facilement l'étiquette «développement durable» sur des programmes ou des initiatives qui ne sont en fait que des interventions mineures destinées à améliorer les systèmes de management environnemental ou l'éco-efficacité. Malgré son nom, le Conseil mondial des entreprises pour un développement durable (WBCSD) a, jusqu'à une date récente, appliqué toute son énergie à promouvoir l'éco-efficacité.

26. Cette critique a été adressée en particulier à l'ISO 14001 (cf. Krut et Gleckman, 1998) et a été formulée d'une manière générale dans certains textes sur la responsabilité sociale des entreprises (cf. Hopkins, 1997).

27. Levy (1997: 60) explique cela par le fait que les grandes entreprises peuvent peut-être davantage se permettre de résister à de coûteux investissements ou à l'inertie bureaucratique. 
Beaucoup de sociétés concentrent étroitement leur attention sur un aspect donné de la responsabilité d'entreprise - la protection de l'environnement par exemple - tout en négligeant d'autres volets tels que les conditions de travail et les droits des populations autochtones. A propos de la certification environnementale, certains syndicats internationaux craignent qu'elle ne légitime les activités d'entreprises qui continuent à violer certains droits fondamentaux. La Fédération internationale des travailleurs du bâtiment et du bois a demandé que la certification des industries forestières tienne désormais compte de critères sociaux empruntés aux normes fondamentales de l'OIT (Development and Cooperation, 1999 : 31). De même, l'Union internationale des travailleurs de l'alimentation et de l'agriculture se montre extrêmement critique à l'égard des producteurs de bananes tels que Chiquita, qui ont adopté le label ECO-OK (par lequel les entreprises concernées s'engagent à utiliser moins de pesticides toxiques). Des organisations et dirigeants syndicaux affirment en effet que Chiquita non seulement continue de nuire à l'environnement mais encore ne respecte pas les droits fondamentaux allant de pair avec la liberté syndicale des travailleurs de pays tels que le Costa Rica (entretien personnel avec une responsable de SITRAP ${ }^{28}$; IUF, 1998).

Le concept de développement durable va bien au-delà de la protection de l'environnement. Il repose sur les principes fondamentaux, énoncés par la Commission mondiale de l'environnement et du développement, de satisfaction des besoins et d'équité intra- et intergénérationnelle (WCED, 1987). On peut, bien sûr, débattre des moyens à mettre en œuvre pour y parvenir. Néanmoins, toute stratégie portant l'étiquette «développement durable» devrait être multidimensionnelle. On a par exemple dit que toute entreprise prenant le développement durable au sérieux devrait être capable d'améliorer sa performance dans six domaines (Welford, 1997): comportement écologique, droits des salariés (droits sociaux et participation des travailleurs) résultats économiques (rentabilité soutenue, création d'emplois et salaires équitables), éthique (codes de conduite, transparence), équité (pratiques commerciales loyales et traitement équitable des partenaires) et éducation (diffusion de l'information et participation à des campagnes). Rares sont les entreprises qui ont essayé d'adopter une stratégie d'une aussi vaste portée.

Outre qu'il laisse de côté des éléments essentiels du concept de développement durable, l' «écologisme d'entreprise» renforce par certains aspects les modes de croissance, de production industrielle, de consommation et de relations NordSud qui sont à la base du développement «non durable». Le label écologique, par exemple, risque de porter préjudice aux pays en développement en constituant un obstacle non tarifaire aux échanges (Markandya, 1997). Selon Dawkins (1995 : 5-6), «l'éco-étiquetage pourrait aggraver la situation actuelle dans laquelle les pays en développement voient s'amenuiser leur part des marchés internationaux et, dans tous les pays, les petites entreprises voient s'amenuiser leur part des marchés nationaux et internationaux. Pour éviter cela, il faudrait que les systèmes d'éco-étiquetage s'accompagnent de mesures énergiques d'action positive destinées à aider les petites entreprises et les exportateurs des pays en développement $»$.

28. Entretien avec Doris Calvo, chef du Département de la condition féminine, SITRAP, avril 1999. 
Selon une étude du secteur international de l'horticulture (UNCTAD et SGS, 1998), des modes de production plus respectueux de l'environnement pourraient être mis en place. Mais cela suppose des investissements, une information et des compétences de gestion qui ne sont généralement pas à la portée des petits producteurs. Résultat: les supermarchés et les importateurs traitent avec le petit nombre de grandes entreprises horticoles, de grandes usines de transformation et de grands exportateurs qui sont mieux organisés et mieux équipés. De ce fait, les petits producteurs se trouvent évincés ou ont une marge de manœuvre réduite (UNCTAD et SGS, 1998 : 7), même si les petits maraîchers conservent des créneaux restreints tels que celui des produits organiques. Parallèlement, en ce qui concerne la responsabilité sociale des entreprises, on peut craindre que beaucoup d'entreprises de pays en développement aient énormément de mal à se conformer aux normes actuellement fixées par les transnationales ou leurs clients. On peut supposer qu'en pareil cas, la notion de responsabilité d'entreprise ne devrait pas se limiter au renforcement des normes mais faciliter l'observation de celles-ci par le fournisseur. En outre, le respect des normes ne devrait pas aggraver d'autres formes de «maldéveloppement» comme cela se produit par exemple lorsque la lutte contre le travail des enfants enfonce davantage les familles dans la pauvreté ou pousse les enfants eux-mêmes dans des formes d'exploitation encore plus préjudiciables.

Les initiatives dues à l'écologisme d'entreprise incitent rarement les consommateurs à changer radicalement leurs habitudes de consommation de façon à contenir efficacement la dégradation de l'environnement. Ainsi, l'étude de cas de l'UITA a montré que le but de la formation dispensée par le fabricant de pesticides était d'apprendre aux gens à utiliser les pesticides classiques d'une façon qui soit moins dangereuse pour la santé et non pas de promouvoir des méthodes alternatives de lutte contre les parasites. Et dans la vie courante, le seul choix qui soit offert aux consommateurs est celui de choisir entre des produits qui ont tous plus ou moins le même impact sur l'environnement; ils ne sont pas encouragés à remettre en cause leurs modes de vie et de consommation (West, 1995 : 19). De même, lorsque des compagnies pétrolières se donnent la peine de dialoguer avec «toutes les parties prenantes», la concertation porte généralement sur la meilleure manière de réaliser un projet donné et non pas sur le bien-fondé de celui-ci (Rowell, 1999).

\section{$\square$ L'arbre qui cache la forêt}

Limiter la réflexion aux initiatives et mesures ponctuelles prises par des entreprises données pour s'acquitter de leur responsabilité sociale et environnementale risque d'occulter la situation d'ensemble créée par les modes d'investissement, de production et de libéralisation économique et par les conséquences de ceux-ci sur l'environnement et la société.

Bien que les chefs d'entreprise les plus engagés dans le mouvement en faveur de la responsabilité d'entreprise aient tendance à proclamer qu'ils n'ont pas un mais trois objectifs, c'est-à-dire un bon bilan financier, certes, mais aussi un bilan environnemental et un bilan social, ils ne consacrent pas moins le gros de leurs investissements à des activités qui ne sortent pas de l'ordinaire (Welford, 1997). Dans une récente interview accordée à $\mathrm{CNN}$, le président de Shell a comparé sa société à un tabouret tenant en équilibre sur trois pieds qui symbolisent trois 
objectifs: financier, environnemental et social. Or le problème est que les trois pieds ne sont absolument pas égaux. A l'évidence, le volume de ressources consacré aux volets environnemental et social n'a rien de comparable avec les sommes investies dans des activités économiques classiques qui engendrent des profits. Il suffit pour s'en rendre compte de jeter un coup d'œil sur le portefeuille de titres de BP Amoco. En 1999, la société a augmenté sa participation dans l'énergie solaire en acquérant Solartex pour 45 millions de dollars (Bruno, 1999), mais a elle a orienté la grande majorité de ses investissements vers l'exploitation de ressources non renouvelables, proposant par exemple d'acheter la compagnie pétrolière ARCO pour environ 31 milliards de dollars (BP Amoco, 1999). Selon le service de presse de Greenpeace, en 1998, pour 10'000 dollars investis dans la prospection et l'exploitation de ressources pétrolières, BP Amoco en a investi 16 dans l'énergie solaire (Media Center, 1999). Dans ces conditions, la comparaison entre les trois objectifs de la société et les trois pieds d'un tabouret n'est-elle pas bancale?

A l'évidence, diverses contraintes structurelles liées à la manière dont est organisée la production vont à l'encontre de la responsabilisation des entreprises. On accuse souvent à cet égard la logique de la production capitaliste, c'est-à-dire la recherche du profit, qui oblige les firmes à réduire ou à externaliser leurs coûts et à s'implanter là où la législation du travail et la réglementation environnementale sont les plus souples. Or le climat de concurrence acharnée découlant de la mondialisation et de la libéralisation ne peut qu'accentuer ce type de contraintes. C'est ainsi que fusions et acquisitions, compressions de personnel, sous-traitance, féminisation et déstructuration de l'emploi, sans compter l'attrait exercé par les havres déréglementés que sont, par exemple, les zones franches d'exportation, permettent à beaucoup de sociétés de réduire leurs effectifs de base et de déplacer leur production vers des sites où la législation est moins regardante en matière de normes écologiques et sociales et où les organisations syndicales sont généralement peu influentes, voire inexistantes.

La tension entre profit et responsabilité est encore plus aigu dans la phase de démarrage des entreprises, celles-ci cherchant alors à rentabiliser rapidement des investissements lourds en externalisant au maximum leurs coûts. Ce problème est mis en évidence dans une étude de l'industrie brésilienne du papier (Carrere, 1999), de laquelle il ressort également que les structures du pouvoir renforcent parfois le processus. Non seulement les grandes entreprises brésiliennes de pâte à papier utilisent leur réseau d'influence pour obtenir des subventions du gouvernement mais encore elles peuvent compter sur l'Etat pour réprimer l'opposition locale suscitée par les externalités. L'analyse de la responsabilité sociale et environnementale des entreprises de cette branche d'activité met aussi en évidence un autre problème structurel lié, celui-ci, à la dimension des entreprises. Même les compagnies qui ont à cœur de protéger l'environnement et de respecter les principes du développement durable ont peu de chances d'y parvenir parce que leurs activités sont d'une ampleur telle que leur impact sur l'environnement est forcément énorme (Carrere, 1999). Dès lors, la question est de savoir si la seule alternative consiste à choisir entre des entreprises très destructrices et d'autres qui le sont moins ou s'il est possible d'envisager un système économique qui s'appuierait sur des entreprises de plus petite taille, plus en phase avec la culture et l'environnement locaux (Carrere, 1999). 
Tenir compte du contexte général signifie aussi tenir compte de la structure des investissements nationaux, régionaux et sectoriels. Il a été démontré qu'au Mexique, par exemple, bien qu'un nombre croissant de firmes prennent désormais des mesures en vue d'améliorer leurs systèmes de management environnemental et que des institutions facilitant cette démarche se créent, le système économique dans son ensemble demeure très pervers (Barkin, 1999). Les investissements sont répartis de telle manière que les industries polluantes s'étendent. En outre, les entreprises ont tendance à s'installer ou à délocaliser dans les régions du pays où la planification et la réglementation sont peu développées. Comme on l'a montré pour l'Inde, le phénomène de la déréglementation compétitive destinée à attirer l'investissement touche non seulement les pays mais aussi les régions et les Etats fédérés d'un même pays (Jha, 1999).

Les structures institutionnelles, notamment dans le cadre de la politique macroéconomique, peuvent aussi réduire le degré de responsabilité sociale et environnementale des entreprises. Tel est le cas en Amérique centrale (Pratt et Fintel, 1999). Les entreprises sont moins tentées d'adopter des mesures de protection de l'environnement lorsque, par exemple, le secteur des services financiers impose des taux d'intérêt élevés et des prêts à court terme. De telles conditions n'incitent pas les entreprises à planifier à long terme, selon les règles de management environnemental. D'autres mesures émanant de ce secteur, par exemple lorsqu'il préconise le recours à tel ou tel montage technique, peuvent encourager les producteurs agricoles à utiliser des technologies obsolètes et nuisibles pour l'environnement. De même, le fisc pénalise l'importation et l'adoption des technologies propres et sous-évalue l'exploitation des ressources naturelles (Pratt et Fintel, 1999).

La responsabilité d'entreprise ne progresse pas de façon linéaire mais par àcoups et parfois, elle tombe carrément dans l'oubli. Tel est évidemment le cas en période de récession, lorsque les entreprises cherchent à réduire leurs coûts par des moyens qui peuvent avoir des effets néfastes sur les plans environnemental et social. Ce phénomène peut aussi être la conséquence d'une rupture de l'équilibre social. Il en est ainsi lorsque les forces sociales et politiques qui œuvrent dans le sens de la déréglementation ou qui se mobilisent pour empêcher toute réglementation prennent le dessus ou lorsque celles qui sont favorables à une forme ou une autre de réglementation s'affaiblissent. La tentative de déstabilisation, voire d'anéantissement, du processus de Kyoto par la Global Climate Coalition en est un bon exemple.

Ainsi, le contexte général lié à l'évolution de la production capitaliste et à la libéralisation de l'économie soulève de graves inquiétudes quant au processus de responsabilisation environnementale et sociale des entreprises. Néanmoins, certains mécanismes et acteurs de la mondialisation pourraient faciliter ce processus.

\section{COMMENT PROMOUVOIR LA RESPONSABILITÉ SOCIALE ET ENVIRONNEMENTALE DES ENTREPRISES?}

La faible ampleur du changement s'explique en partie par la jeunesse d'un mouvement qui n'a véritablement pris son essor que dans les années 90 . Il convient par conséquent de voir s'il existe des forces ou un climat propices à une intensification des initiatives favorables à la responsabilisation des entreprises. Et là, il 
y a des raisons d'espérer car certains facteurs relativement contraignants œuvrent dans ce sens. Nous en relèverons trois.

Premièrement, certaines entreprises considèrent qu'elles ont intérêt du point de vue économique à améliorer leur performance et leur image sur les plans environnemental et social pour se placer sur les marchés des produits et services «verts» ou «éthiques » et être plus compétitives. Deuxièmement, la mondialisation a notamment eu pour effet d'élargir l'offre à l'échelle mondiale. Il est donc normal que les dirigeants des multinationales de certaines branches veillent à ce que leurs filiales et leurs fournisseurs respectent des normes minima en matière de protection de l'environnement et de conditions de travail afin de préserver l'image de l'entreprise ou de sa production, la valeur de ses actions et ses parts de marché. Voila pourquoi certaines STN tiennent à ce que leurs filiales et leurs sous-traitants adhèrent à des codes de conduite ou obtiennent une certification écologique. Troisièmement, et c'est peut-être le facteur le plus important, un mouvement social d'envergure mondiale et relativement puissant vient de naître, qui exige davantage de transparence de la part des entreprises. Ce mouvement, qui est particulièrement influent dans les pays industrialisés riches attire l'attention sur certaines STN en les obligeant à changer de pratique.

Bien que ces «moteurs» soient de nature à inciter les entreprises à mieux tenir compte des préoccupations relatives à l'environnement et aux questions sociales, le changement demeurera probablement aléatoire, de portée inégale selon les sociétés, les pays et les branches d'activité et, pour ce qui est du développement durable, chargé de contradictions. Cet état de choses n'est pas uniquement dû à l'origine récente de cette mutation mais aussi à la façon dont les entreprises choisissent de réagir aux contraintes économiques, politiques et structurelles - réaction qui consiste à présenter une image acceptable et à aménager superficiellement les modes et pratiques de gestion plutôt qu'à orienter radicalement les stratégies industrielles et commerciales vers le respect des règles sociales et de l'environnement.

Face à cette relative inefficacité des ressorts actuels de la responsabilité sociale et environnementale des entreprises, comment intensifier et «ancrer» le mouvement afin d'amener les entreprises à contribuer davantage à un développement durable? L'approche institutionnelle actuellement dominante privilégie les «initiatives volontaires » et le «partenariat».

Les initiatives volontaires englobent toute une série de mesures qui vont au-delà de la législation en vigueur sur la défense de l'environnement et la protection sociale. Elles sont souvent considérées comme étant plus efficaces que des dispositions réglementaires en ce sens que leurs objectifs sont plus ambitieux, qu'elles engendrent moins de frais d'administration et d'exécution et qu'elles sont applicables plus rapidement (UNEP, 2000). Mais elles présentent aussi des inconvénients.

Comme nous l'avons vu plus haut, le point faible des initiatives volontaires réside souvent dans le fait que leur conception ne tient compte ni des principes de transparence, de vérification indépendante et de participation des travailleurs et de la collectivité ni des normes internationales qui régissent la protection des travailleurs, de l'environnement et des droits de l'homme. En outre, des initiatives telles que les codes de conduite et les systèmes de certification émanent de gouvernements, d'ONG ou d'entreprises du Nord. Bien souvent, les gouverne- 
ments et les ONG du Sud ne sont pas consultés à propos de décisions qui les concernent.

Le problème le plus évident est qu'un nombre limité seulement de sociétés adopteront de telles initiatives. A la différence des lois, ces initiatives ne comportent généralement aucun mécanisme contraignant les entreprises qui ne le font pas à se conformer à des normes plus strictes. L'efficacité de nombreuses initiatives volontaires dépend du contexte institutionnel, c'est-à-dire, entre autres, de l'existence de lois sur la divulgation et la liberté de l'information, d'organismes de surveillance et d'une forte mobilisation de la société civile. Dans beaucoup de pays, ces conditions ne sont que partiellement réunies, voire inexistantes.

Ayant évalué certaines formes d'initiatives volontaires, l'OCDE a conclu que celles-ci n'étaient pas d'une réelle efficacité en ce qui concerne la protection de l'environnement mais qu'elles pouvaient être d'une certaine utilité sur les plans de l'information et de la sensibilisation (OECD, 1999b). Outre les problèmes dus à l'absence de contrôle, le rapport de l'OCDE met en garde contre le risque de «détournement du pouvoir réglementaire», les entreprises pouvant utiliser les initiatives volontaires pour empiéter sur l'action des pouvoirs publics.

Les partenariats entre entreprises et ONG ou organisations multilatérales sont généralement justifiés par la nécessité, en période de mondialisation et d'interdépendance économique, d'établir de nouvelles formes de gouvernance faisant appel à la collaboration de tous les acteurs concernés. Non seulement les gouvernements n'ont pas le pouvoir de décider de l'orientation du développement économique mais encore leurs prérogatives sont à bien des égards affaiblies. Dans ces conditions, nous dit-on, le secteur privé, la société civile, les institutions multilatérales et les pouvoirs publics doivent s'associer en constituant des partenariats, «seule manière de résoudre les questions complexes et interdépendantes par la coopération et la participation au sein d'un même pays et entre les nations (Nelson, 1996 : 13).

Cette démarche en apparence pragmatique, constructive et coopérative est très séduisante. Elle l'est d'ailleurs tellement que beaucoup d'organisations se précipitent tête baissée dans des "partenariats» avec le monde des affaires sans se poser trop de questions sur les conséquences. Il est à cet égard particulièrement inquiétant de constater la légèreté des critères sur lesquels se fondent souvent les Nations Unies et d'autres organisations pour choisir leurs partenaires du privé. Egalement préoccupants sont, d'une part, la manière d'étouffer les critiques exprimées face au rapprochement des $\mathrm{ONG}$ et des institutions des Nations Unies avec le milieu des entreprises et, d'autre part, le problème du «détournement institutionnel» causé par l'influence que finit par exercer le monde des affaires sur les décisions d'intérêt général. (Utting, 2000b).

L'aspect le plus critiquable des initiatives volontaires et des partenariats est que ces deux démarches affaiblissent les principaux ressorts de la responsabilisation des entreprises, à savoir la réglementation par les pouvoirs publics, la négociation collective et certaines formes de militantisme de la société civile. Si l'on examine la genèse de la notion de responsabilité sociale et environnementale des entreprises et de certaines réformes contemporaines des politiques et pratiques des entreprises, on retrouve toujours au moins un de ces facteurs. 
Les initiatives volontaires sont trop souvent considérées comme étant indépendantes de la réglementation des pouvoirs publics ou comme une solution de rechange à cette réglementation. En réalité, elles trouvent souvent leur origine dans diverses formes de législation et de sanction de l'Etat, dont dépend leur réussite. Elles sont en général plus efficaces lorsqu'elles sont d'une manière ou d'une autre associées à une réglementation et à un contrôle indépendant (UNEP, 2000).

Pour que la responsabilité sociale et environnementale des entreprises progresse, le mouvement de revendication de la transparence des entreprises doit absolument se renforcer. Ce mouvement place certaines sociétés sous les projecteurs, recense et fait connaître les pratiques incorrectes, menace la réputation des entreprises et exige d'elles qu'elles améliorent leur bilan social et environnemental. Certains secteurs du monde des affaires sont obligés de tenir compte de telles pressions mais certaines entreprises le font par le biais de changements fragmentaires de leur politique et de leurs pratiques.

En l'absence de réglementation gouvernementale et internationale et d'une pression plus concertée, mieux coordonnée, de la société civile, la promotion de la responsabilité sociale et environnementale des entreprises dans les pays en développement restera, au mieux, tiède. L'analyse des moteurs du changement qui précède donne à penser que les STN et autres grandes entreprises continueront sans doute à adopter des mesures relatives à la responsabilité sociale et environnementale. A noter à cet égard que les changements de comportement des entreprises ne sont pas que de la poudre aux yeux ou des relations publiques comme l'affirment certains observateurs. Cependant, les mesures prises risquent de ne représenter que le minimum acceptable et d'être inégales. En facilitant le bon fonctionnement de la production et du commerce, et souvent en diluant les méthodes alternatives de changement, de telles mesures risquent fort de favoriser davantage la croissance économique et la stabilité du capitalisme que le développement durable.

\section{BIBLIOGRAPHIE}

Annan, K. (1999), «A compact for the new century », Address to the World Economic Forum, Davos, 31 January (www.un.org/partners/business/davos.htm).

Barber, J. (1998), «Responsible action or public relations? NGO perspectives on voluntary initiatives », Industry and Environment, Vol. 21, No. 1-2, January-June, pp. 19-22.

Barkin, D. (1999), The Greening of Business in Mexico, Discussion Paper No. 110, UNRISD, Geneva.

Bendell, J. (ed.) (1998), «Business-NGO relations and sustainable development», Greener Management International: The Journal of Corporate Environmental Strategy and Practice, Issue 24, winter.

Bennett, T. (1986), «Introduction: Popular culture and 'the turn to Gramsci' », in T. Bennett, C. Mercer and J. Woollacott (eds.), Popular Culture and Social Relations, Open University Press, Milton Keynes.

BP Amoco (1999), Shareholder Documents: Listing Particulars, (www.bpamoco.com/investors/arco/ egm.htm).

Bruno, K. (1999), «Summer greenwash award: BP Amoco's 'Plug in the Sun' program», in Corporate Watch Greenwash Awards, (www.corpwatch.org/trac/greenwash/bp.html).

BUSCO (1995), Contribution of Corporations to Social Development, Business Association for the World Social Summit, Paris.

Carrere, R. (1999), The Environmental and Social Effects of Corporate Environmentalism in the Brazilian Market Pulp Industry, mimeo, UNRISD, Geneva (proposed chapter in P. Utting (ed.), op.cit.).

Chatterjee, P. and M. Finger (1994), The Earth Brokers: Power, Politics and World Development, Routledge, London.

Clean Clothes Campaign (1999) (www.cleanclothes.org/codes/inilivingwa.htm). 
Colchester, M. (1990), «The international tropical timber organization: Kill or cure for the rainforests ?", The Ecologist, Vol. 20, No. 5, pp. 166-173.

Corporate Watch (1996) (www.oneworld.org).

CSD (1999), Voluntary initiatives and agreements: Report of the Secretary-General, United Nations Economic and Social Council, E/CN.17/1999/12, 5 April.

Currah, K.R. (1999), Activating the Self-correcting Mechanism of Civil Society, mimeo, World Vision, Milton Keynes.

Dawkins, K. (1995), Ecolabelling: Consumer's Right to Know or Restrictive Business Practice?, mimeo, Institute for Agriculture and Trade Policy, Minneapolis.

Development and Cooperation (1999), «Certification to save the world's forests », D+C, No 2.

Dommen, E. (1999), Pertinence et limites des codes éthiques, mimeo, Geneva.

Evans, P. (1998), «Transnational corporations and Third World states: From the old internationalization to the new ", in Kozul-Wright and Rowthorn (eds.), op cit.

FAO (1997), State of the World's Forests, 1997, FAO, Rome.

Flaherty, M. and A. Rappaport (1991), Multinational Corporations and the Environment: A Survey of Global Practices, The Center for Environmental Management, Tufts University, Medford.

-, and A. Rappaport (1999), Corporate Environmentalism: From Rhetoric to Results, mimeo, UNRISD, Geneva (proposed chapter in P. Utting (ed.), op.cit.).

Forcese, C. (1996), Commerce with Conscience? Human Rights and Business Codes of Conduct, International Centre for Human Rights and Democratic Development, Montreal.

Forgacs, D. (1988), An Antonio Gramsci Reader: Selected Writings, 1916-1935, Schocken Books, New York.

Fortune (1999), 1999 Global 5 Hundred, Vol. 140, No. 3, August.

Frankel, C. (1999), «One foot in the future», Tomorrow, Vol. IX, No. 1, January/February, pp. 11-12.

Freeman, R. (1984), Strategic Management: A Stakeholder Approach, Pitman, Boston.

FSC (1999)(www.fscoax.org).

Gallin, D. (1999a), Organized Labour as a Global Social Force, paper presented at IR2 Workshop, Washington, DC, 20 February 1999.

-, (1999b), Trade Unions and NGOs in Social Development: A Necessary Partnership, mimeo, UNRISD, Geneva.

Gereffi, G. (1994), «Capitalism, development and global commodity chains », in L. Sklair (ed.), Capitalism and Development, Routledge, London.

-, M. Korzeniewicz and R. Korzeniewicz (1994), «Introduction: Global commodity chains», in Gereffi and Korzeniewicz (eds.), Commodity Chains and Global Capitalism, Greenwood Press, London.

Gonçalves, M.T. et al. (1994), Exploraçao florestal no norte do ES e sul da Bahia: Impactos e alternativas, FASE/IBASE, Rio de Janeiro.

Greer, J. and K. Bruno (1996), Greenwash: The Reality Behind Corporate Environmentalism, Third World Network, Penang.

Hagen, K. (1998), «Issues involving codes of conduct from an ILO perspective», in A.Wild, op.cit.

Hanks, J. (1999), Promoting Corporate Environmental Responsibility: What Role for 'Self-regulatory' and 'Co-regulatory' Policy Instruments?, mimeo, UNRISD, Geneva (proposed chapter in P. Utting (ed.), op.cit.).

Hansen, M. (1999a), Environmental Regulation of Transnational Corporations: Needs and Prospects, mimeo, UNRISD, Geneva (proposed chapter in P. Utting (ed.), op.cit.).

-, (1999b), Environmental Management in Transnational Corporations in Asia: Does Foreign Ownership Make a Difference? Preliminary Results of a Survey of Environmental Management Practices in 154 TNCs, Occasional Paper, CBS/UNCTAD Cross Border Environmental Management Project, Copenhagen Business school, Copenhagen.

Harverson, P. and R. Corzine (1997), «In defence of international reputations », Financial Times, 31 October, p. 15 .

Hawken, P. (1993), A Declaration of Sustainability, September/October, Utne Reader.

Henderson, H. (1996), Building a Win-Win World, Berrett-Koehler Publishers, San Francisco.

Hirst, P. and J. Zeitlin (1991), «Flexible specialisation versus post-Fordism: Theory, evidence and policy implications », Economy and Society, Vol. 20, No.1, February, pp. 1-56.

Hopkins, M. (1997), «Defining indicators to assess socially responsible enterprises», Futures, Vol. 29, No. 7, pp. 581-603.

Hurst, P. (1999), IUF Case Study: The Global Pesticide Industry's «Safe Use and Handling " Training Project in Guatemala, International Union of Food and Agricultural Workers, Geneva.

IFAT (1999), The Business of Fair Trade: Livelihoods, markets and sustainability, conference report, IFAT, Oxford. 
IIED (1996), Towards a Sustainable Paper Cycle, IIED/WBCSD, London/Geneva.

ILO, Sectoral Activities Programme (1999), Voluntary initiatives affecting training and education on safety, health and environment in the chemical industries, ILO, Geneva.

ISO (1998), ISO 14000: Meet the Whole Family!, International Organization for Standardization, Geneva.

—, (1999), The ISO Survey of ISO 9000 and ISO 14000 Certificates. The eighth cycle - 1998, International Organization for Standardization, Geneva.

IUF (1998), «International banana conference explores routes 'towards a sustainable banana economy'», IUF News Bulletin, Nos. 3 and 4, International Union of Food and Agricultural Workers, Geneva.

Jeanrenaud, S. (1998), Can the Leopard Change its Spots? Exploring People-Oriented Conservation within the WWF, doctoral thesis, University of East Anglia, Norwich.

Jha, V. (1999), Investment Liberalization and Environmental Protection: Conflicts and Compatibilities in the Case of India, Occasional Paper, CBS/UNCTAD Cross Border Environmental Management Project, Copenhagen Business School, Copenhagen.

Kasperson, R., R.Kates, C. Honenemser and J. Kasperson (1988), Corporate Management of Health and Safety Hazards : A Comparison of Current Practice, Westview, Boulder.

Kolk, A., R. van Tulder and C. Welters (1999), «International codes of conduct and corporate social responsibility: Can transnational corporations regulate themselves?», Transnational Corporations, Vol. 8, No. 1, April, pp. 143-180.

Kolodner, E. (1994), Transnational Corporations: Impediments or Catalysts of Social Development?, Occasional Paper No. 5, World Summit for Social Development, UNRISD, Geneva.

Kozul-Wright, R. and B. Rowthorn (1998), «Introduction: Transnational corporations and the global economy», in Kozul-Wright and Rowthorn (eds.), Transnational Corporations and the Global Economy, Macmillan, London.

Krut, R. (1997), Globalization and Civil Society: NGO Influence in International Decision-making, Discussion Paper No. 83, UNRISD, Geneva.

—, and H. Gleckman (1998), ISO 14001: A Missed Opportunity for Sustainable Global Industrial Development, Earthscan, London.

Levy, D. (1995), «The Environmental Practices and Performance of Transnational Corporations », Transnational Corporations, Vol. 4, No. 1, April, pp .44-67.

—, (1997), «Environmental management as political sustainability», Organization \& Environment, Vol. 10, No.2, June, pp. 126-147

Markandya, A. (1997), «Eco-labelling: An introduction and review», in S. Zarrilli, V. Jha and R. Vossenaar (eds.), Eco-Labelling and International Trade, Macmillan, London.

Media Center (1999), «We laughed! We cried! But mostly we cried!», Press Releases (www.greenpeaceusa.org/media/press_releases/99_4_22text.htm).

Murphy, D. and J. Bendell (1999), Partners in Time? Business, NGOs and Sustainable Development, Discussion Paper No. 109, UNRISD, Geneva.

Nelson, J. (1996), Business as Partners for Development: Creating Wealth for Countries, Companies and Communities, The Prince of Wales Business Forum, London.

NEF and CIIR (1997), Open Trading: Options for Effective Monitoring of Corporate Codes of Conduct, NEF/CIIR, London.

Novartis (1998), Report of the Novartis Foundation for Sustainable Development 1997/1998, Novartis Foundation for Sustainable Development, Basel.

OECD (1999a), Codes of Corporate Conduct: An Inventory, OECD, Paris.

-, (1999b), Voluntary Approaches for Environmental Policy: An Assessment, OECD, Paris

O’Neill, K. (1999), Internetworking for Social Change: Keeping the Spotlight on Corporate Environmentalism, Discussion Paper No. 111, UNRISD, Geneva.

Porter, M. and C. van der Linde (1995), «Green and competitive: Ending the stalemate», Harvard Business Review, September-October, pp. 120-134.

Pratt, L. and E. Fintel (1999), Environmental Management as an Indicator of Business Responsibility in Central America, mimeo, UNRISD, Geneva (proposed chapter in P. Utting (ed.), op.cit.).

Richter, J. (forthcoming), The Regulation of the Infant Food Industry (provisional title).

Roddick, A (1998) «Profiting the poor» Developments, Issue 2, Second Quarter, pp. 5-7.

Rodríguez, S. and M.A. Camacho (1999), Bioprospecting in Costa Rica: Facing New Dimensions of Social and Environmental Responsibility, mimeo, UNRISD, Geneva (proposed chapter in P. Utting (ed.), op.cit.).

Romeijn, P. (1999), Green Gold: On Variations of Truth in Plantation Forestry, Treebook 2, Treemail Publishers, Heelsum, the Netherlands.

Rowell, A. (1999), «Greenwash goes legit», Guardian, 21 July, p.5. 
Sargent, C. and S. Bass (eds.) (1992), Plantation Politics: Forest Plantations in Development, Earthscan, London.

Schmidheiny, S. (1992), Changing Course: A Global Business Perspective on Development and the Environment, MIT Press, Cambridge, Massachusetts.

-, R. Chase and L. DeSimone (1997), Signals of Change: Business Progress towards Sustainable Development, WBCSD, Geneva.

_, and F. Zorraquín, with WBCSD (1996), Financing Change: The Financial Community, Eco-Efficiency, and Sustainable Development, MIT Press, Cambridge, Massachusetts.

Schrempp, J. (1997), «Corporate culture and a community contribution», Financial Times, 31 October, p.15.

Shiraz A. (1998), «Social Reporting», Australian CPA, November.

Stigson, B. (1997), «The Business Charter for Sustainable Development», in F. Dodds (ed.), The Way Forward: Beyond Agenda 21, Earthscan, London.

—, (1999), «Business is shaping the agenda», in Tomorrow, Vol. IX, No. 4, July/August, p. 45.

Tesner S. (1999), The United Nations and Business: A Partnership Recovered, St. Martin's Press, New York.

The Concise Oxford English Dictionary (1999), Oxford University Press, Oxford.

ToBI (1997), NGO Perspective on Responsible Entrepreneurship(www.coopamerica.org/isf/tobi/respentr/NGO\%20perspectives.html).

Thomson, B. (1998), Fair Trade - Frequently Asked Questions - FAQ, (www.web.net/fairtrade/who/ fair2.html).

Tomorrow (1994), «In search of environmental excellence», Vol. IV, No. 4, July/August.

TRAC (1999), A Perilous Partnership: The United Nations Development Programme's Flirtation with Corporate Collaboration, Transnational Resource and Action Center, San Francisco.

Traidcraft (1998), Fair Trade and Ethical Trade: Distinct but Complementary (www.web.net/fairtrade/other/briefing.html).

UNCTAD (1996), Self-Regulation of Environmental Management: An Analysis of Guidelines Set by World Industry Associations for their Member Firms, UNCTAD, Geneva.

—, (1998), World Investment Report 1998: Trends and Determinants, UNCTAD, Geneva.

—, (1999), World Investment Report 1999 : Foreign Direct Investment and the Challenge of Development, UNCTAD, Geneva.

-, (2000), World Investment Report 2000 : Cross-border Mergers and Acquisitions and Development, UNCTAD, Geneva.

-, and SGS (1998), International Market Access Information: Horticultural Sector, United Nations, Geneva.

UNDP (1998), Human Development Report, 1998, Oxford University Press, Oxford.

—, (1999a), The Global Sustainable Development Facility: Project Description, mimeo, UNDP, New York.

-, (1999b), «Multinational corporations join discussions with UNDP to establish global sustainable development facility », UNDPFlash, Monday 15 March (www.undp.org/flash/fleng.htm).

UNEP (1994), Company Environmental Reporting: A Measure of the Progress of Business \& Industry Towards Sustainable Development, SustainAbility/UNEP Industry and Environment, London/Paris.

—, (1998), «Voluntary initiatives for responsible entrepreneurship: A question and answer guide», Industry and Environment, Vol. 21, No.1-2, January-June, pp. 4-9.

-, (2000), Voluntary Initiatives: Current Status, Lessons Learnt and Next Steps, UNEP, Paris

_, and SustainAbility (1997), Engaging Stakeholders: The 1997 Benchmark Survey, SustainAbility, London

United Nations (1999), Business and the UN: Partners in Sustainable Development, United Nations, New York.

UNRISD (1995), States of Disarray: The Social Effects of Globalization, UNRISD, Geneva, distributed by Earthscan, London.

-, and UNA (1998), Business Responsibility for Environmental Protection in Developing Countries: Report of the International Workshop (Heredia, Costa Rica, 22-24 September 1997), UNRISD, Geneva.

UN Wire, «Business Humanitarian Forum: NGOs Criticize Ogata, Bellamy», United Nations Foundation UN Wire, 29 September.

(www.unfoundation.org/unwire/archives/UNWIRE990929.cfm).

Utting, P. (1992), Economic Reform and Third-World Socialism: A Political Economy of Food Policy in Post-Revolutionary Societies, Macmillan, London. 
—, (1993), Trees, People and Power: Social Dimensions of Deforestation and Forest Protection in Central America, Earthscan, London.

—, (2000a), Business Responsibility for Sustainable Development, OPG 2, UNRISD, Geneva.

-, (2000b), UN-Business Partnerships: Whose Agenda Counts?, paper presented at «Partnerships for Development or Privatization of the Multilateral System?» seminar, North-South Coalition, Oslo, Norway, 8 December, 2000.

-, (ed.) (forthcoming), The Greening of Business in the South: Rhetoric, Practice and Prospects.

von Moltke K. et al. (1998), Global Product Chains: Northern Consumers, Southern Producers and Sustainability, Environment and Trade 15, United Nations Environment Programme, Geneva.

Watts, P. and Lord Holme (1999), Corporate Social Responsibility: Meeting Changing Expectations, World Business Council for Sustainable Development, Geneva.

Welford, R. (1997), Hijacking Environmentalism: Corporate Responses to Sustainable Development, Earthscan, London.

—, and D. Jones (1996), «Beyond environmentalism and towards sustainable organization», in R. Welford (ed.) Corporate Environmental Management: Systems and Strategies, Earthscan, London.

West, K. (1995), «Ecolabels: The industrialization of environmental standards», The Ecologist, Vol. 25, No. 1, January/February, pp. 16-20.

Wild, A. (1998), A Review of Corporate Citizenship and Social Initiatives: Social Citizenship - What's Going On ... and Why?, Enterprise and Cooperatvie Development Department, ILO, Geneva.

Wood, D.J. (1991), "Social issues in management: Theory and research in corporate social performance », Journal of Management, Vol. 17, pp. 383-406.

World Bank (1998), World Development Report 1998, Oxford University Press, Oxford.

—, (1999), 1999 World Development Indicators, World Bank, Washington, DC.

WBCSD (1999), «Why is CSR climbing on the international agenda», Tomorrow, Vol. IX, No. 3, May/June.

WCED (1987), Our Common Future, Oxford University Press, Oxford/New York.

World Rainforest Movement (1999), Tree Plantations: Impacts and Struggles, World Rainforest Movement, Montevideo.

World Resources Institute (1998), World Resources Institute and General Motors Open Dialogue on Global Climate (www.icg.org/wri/press/wrigm-nr-html).

Zarrilli, S., V. Jha and R. Vossenaar (eds.) (1997), Eco-Labelling and International Trade, Macmillan, London. 